\title{
SIMULATION OF HIGH-FREQUENCY WAVE PROPAGATION IN COMPLEX CRUSTAL WAVEGUIDES USING GENERALIZED SCREEN PROPAGATORS
}

\author{
Ru-Shan Wu, Xian-Yun Wu AND XIAO-Bi XIE \\ Modeling and Imaging Laboratory, Institute of Geophysics and Planetary Physics, University of \\ California, Santa Cruz, California, USA
}

\begin{abstract}
In the crustal waveguide environment, the major part of wave energy is carried by forward propagating waves, including forward scattered waves. Therefore, neglecting backscattered waves in numerical modeling will not modify the main features of regional waves in most cases. By neglecting backscattering in the theory, the wave modeling becomes a forward marching problem in which the next step of propagation depends only on the present values of the wavefield in a transverse cross-section and the heterogeneities between the present cross-section and the next one (wavefield extrapolation interval). The saving of computation time and computer memory is enormous. A half-space screen propagator (generalized screen propagator) has been developed to accommodate the free-surface boundary condition for modeling $\mathrm{SH}$ wave propagation in complex crustal waveguides. The $\mathrm{SH}$ screen propagator has also been extended to handle irregular surface topography using conformal or nonconformal topographic transforms. The screen propagator for modeling regional $\mathrm{SH}$ waves has been calibrated extensively against some full-wave methods, such as the wavenumber integration, finite-difference and boundary element methods, for different crustal models. Excellent agreement with these full-wave methods demonstrated the validity and accuracy of the new one-way propagator method. For medium size problems, the screen-propagator method is $2-3$ orders of magnitude faster than finite-difference methods. It has been used for the simulation of $\mathrm{Lg}$ propagation in crustal models with random heterogeneities and the related energy partition, attenuation and blockage. It is found that the leakage attenuation of $\mathrm{Lg}$ waves caused by large-angle forward scattering by random heterogeneities, which scatters the guided waves out of the trapped modes and leaking into the mantle, may contribute significantly to Lg attenuation and blockage in some regions. In the case of P-SV elastic screen propagators, plane wave reflection calculations have been incorporated into the elastic screen method to handle the free surface. Body waves, including the reflected and converted waves, can be calculated by real wavenumber integration; while surface waves (Rayleigh waves) can be obtained with imaginary wavenumber integration. Numerical tests proved the validity of the theory and methods.
\end{abstract}

Keywords: Lg-wave, Crustal wave guide, One-way propagator, Seismic wave scattering 


\section{INTRODUCTION}

High-frequency regional wave propagation in complex crustal waveguides is one of the most challenging problems in theoretical and computational seismology. A good understanding of propagation, scattering, attenuation and wave-type conversion of regional waves and the availability of analytical/numerical tools to simulate and analyze these phenomena for complex crustal structures, including rough surface, Moho topography and small-scale heterogeneities, are crucial to the applications of regional waves to various geophysical problems. Regional wave tomography for crustal structures, path correction for discrimination and yield estimation of low-yield nuclear tests, location determination of earthquakes, or underground explosions using regional phases are examples among the possible applications. Nuclear explosion monitoring at regional distances is even more demanding for the simulation and analyzing tools. For this purpose, simulation algorithms are desirable for generating synthetic waveforms for high frequencies up to $25 \mathrm{~Hz}$ at distances greater than $1000 \mathrm{~km}$.

Substantial efforts have been made in modeling regional wave propagation. Methods based on layered earth models, such as the reflectivity and mode summation methods (e.g., Bouchon et al., 1985; Kennett, 1989, 1990; Maupin, 1989; Baumgardt, 1990; Campillo, 1990; Campillo and Paul, 1992; Campillo et al., 1993; Gibson and Campillo, 1994) have very high efficiency and can be applied to relatively high frequencies, but they can be used only for very simplified cases with layered or smoothly varying layered models, or be applied to part of the wavefield. Modeling techniques that can treat realistic 3-D heterogeneous media, rather than smoothly varying layered media, are needed to test and study many observations and hypotheses. New modeling methods are needed to handle sudden changes of crustal thickness, strong lateral variations and irregular 3-D heterogeneities. As pointed out by Campillo et al. (1993), actual Lg amplitudes are reduced more than 10 times for paths passing through an anomalous zone on the east side of the Alpine range, while the modeling results using existing methods (including the effect of known large-scale lateral structural variation) only account for $20-30 \%$ of the amplitude reduction. Other attenuation mechanisms such as the scattering and attenuation by small-scale heterogeneities must be taken into account.

Kennett (1984, 1998) and Maupin and Kennett (1987) developed a coupled mode method for calculating guided seismic waves in horizontally varying structures. The method works well for relatively low frequency waves in moderately heterogeneous models (for a summary of the coupling mode method, see Chapter 2 of this book by Maupin). However, the implementation of the method for high frequency 3-D models still requires formidable computational efforts.

Chen (1990, 1995) developed a global R/T (Reflection/Transmission) matrices method, for simulating the seismic wave excitation and propagation in an arbi- 
trarily multi-layered medium with irregular interfaces, which can be regarded as an extension of the generalized R/T coefficients method (reflectivity method) for the horizontally layered case by incorporating the T-matrix approach (for a summary, see Chapter 4 of this book by Chen). Again the application of the method is limited to low frequencies and short propagation distances.

Cormier and Anderson (1996, 1997) applied elastic Born scattering (in the regime of Rayleigh scattering) to the locked-mode solution for plane layered media to calculate the effects of small-scale heterogeneities. However, the approximation is limited to single scattering and is only good for heterogeneities with scales much smaller than the wavelength. Ray method has very limited success in modeling regional waves due to the chaotic behavior of rays caused by the multiple reflections from the free-surface and Moho. Keers et al. (1996a, 1996b) applied the Maslov integral method to avoid the caustics and pseudocaustics (caustics of plane waves) by working in the phase-space. However, when chaos develops in the ray system, more complicated caustics arise for which the Maslov method does not work. In addition, ray-tracing computation is very timeconsuming in this case. An alternative and flexible approach using ray-tracing has been developed by Kennett (1986), Bostock and Kennett (1990) and Kennett et al. (1990), in which ray diagrams are used to study Lg waves crossing structural boundaries. The method agrees well with modal calculations and can be applied to surface topography, 3-D crustal structures and other cases. However, the method cannot provide information on wave phenomena for complicated waveguides.

Finite-difference methods (e.g., Xie and Lay, 1994; App et al., 1996; Goldstein et al., 1996, 1997, 1999; Husebye and Ruud, 1996; Jih, 1996; Nolte et al., 1996; Jones et al., 1997; McLaughlin and Wilkins, 1997; Bradley and Jones, 1998, 1999; Xie et al., 2005) and pseudo-spectral methods (e.g., Kosloff et al., 1990; Archambeau et al., 1996; Schatzman, 1996; Furumura and Kennett, 1997) are commonly-used numerical methods that have been extensively applied to regional wave propagation. Theoretically, these methods can deal with arbitrarily heterogeneous media. However, it is necessary to use very dense spatial sampling to avoid grid dispersion for long distance regional wave propagation (for grid dispersion problem, see Fornberg, 1987). The capability of the presentday computers usually restricts them to short propagation ranges and relatively low frequencies, which prevents them from being applied to more realistic cases.

The state-of-the-art of the traditional simulation techniques for regional waves has its application to relatively low frequencies and short propagation distances. Correspondingly, the volume heterogeneities and surface irregularity in the crustal models are limited to rather large scales. However, high-frequency regional waves up to $20 \mathrm{~Hz}$ or higher have been observed over different distances, ranging from a few hundred kilometers to more than one thousand kilometers (e.g., Ni et al., 1996; Herrmann et al., 1997; Lay et al., 1999). Since high-frequency waves 
can be used for event locations with high accuracy, simulation and modeling of high-frequency regional wave propagation are very desirable for many applications. For high-frequency wave propagation, scattering and attenuation, the role of small-scale heterogeneities and surface roughness are all important.

The existence of small-scale heterogeneities in the crust and the associated seismic wave scattering have been known among seismologists (e.g., Aki and Richards, 1980; Wu and Aki, 1988, 1989, 1990; Sato and Fehler, 1998). However, the effects of these heterogeneities on guided wave $(\mathrm{Lg})$ propagation in the crust have not been explored extensively. The reasons may be the following. First, the spectra, strength and distribution of the small-scale heterogeneities in different regions are not well-known. Very few data sets can be used to characterize the paths concerned. Second, there lack analytical and numerical tools to model or analyze their influence on the guided wave propagation. The theory of wave propagation in unbounded random media has been well developed. However, for waves in complex crustal waveguides with random heterogeneities, the theoretical difficulties are overwhelming, and no analytical tools are available for performing realistic calculations. Therefore, numerical methods for simulating regional wave propagation in complex waveguides with small-scale heterogeneities are highly desirable. It has become clear that small-scale heterogeneities are widely distributed in tectonically active regions. Strong topographic variation is the manifestation of tectonically active regions and often the indication of small-scale heterogeneities. Figure 1 gives a topographic profile (top panel) and its power spectrum (bottom panel) for a path crossing the Tibet region. The slope of the spectral rolloff is close to $1 / k$, a flicker noise spectrum, very rich in small-scale variations. This spectrum is similar to the observations of the sonic well-log in the KTB super-deep continental drilling well (Wu et al., 1994; Jones and Holliger, 1997; Goff and Holliger, 1999), where the spectrum also has a $1 / k$ slope. Recently, Goff and Holliger (1999) explained the $1 / k$ spectra as a combination of hierarchical, multi-scale heterogeneities. Overall, the $1 / k$ spectra demonstrate the richness of small-scale heterogeneities.

Recently, the generalized screen method has been introduced into seismic wave simulations and applied to the problems of both exploration and theoretical seismology. The generalized screen method is based on the one-way wave equation and the one-return approximation. The one-way generalized screen propagator (GSP) neglects backscattered waves, but correctly handles all the forward multiple-scattering effects, e.g., focusing/defocusing, diffraction, interference, and conversion between different wave types. The one-return approximation is also called the De Wolf approximation (De Wolf, 1971, 1985), which neglects the reverberation between screens and can simulate multiple-forescattering-singlebackscattering (MFSB). Significant progress has been made on the development of an elastic complex screen (ECS) method for modeling elastic wave propagation and scattering in arbitrarily complicated structures (Wu, 1994, 1996; Xie and Wu, 1995, 2001; Wild and Hudson, 1998; Wu and Wu, 1999). The method is two 

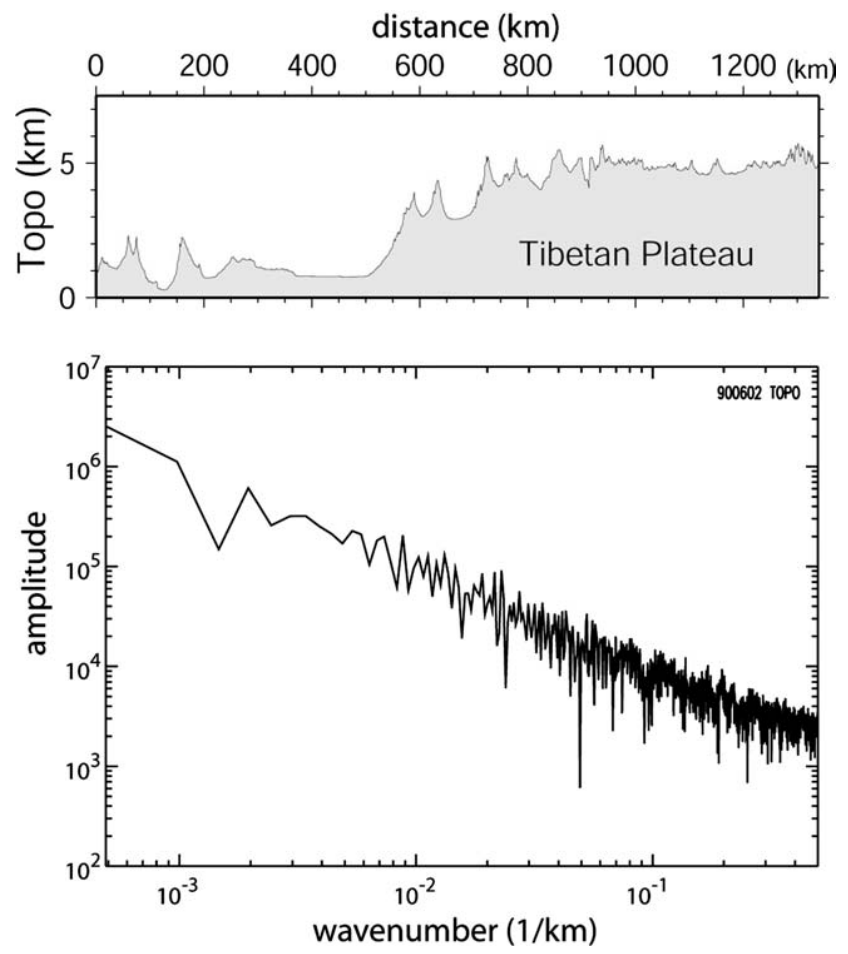

FIG. 1. Topographic profile (top) and its power spectrum (bottom) for a path crossing the Tibet region.

to three orders of magnitude faster than the elastic finite-difference method for a medium sized 3-D problem. For detailed derivation and the physical meaning of the MFSB approximation and the generalized screen method, see Chapter 5 of this volume. The screen method has been successfully used in forward modeling (Wu, 1994; Wu and Huang, 1995; Wu et al., 1995; Xie and Wu, 1995, 1996, 1999, 2001; $\mathrm{Wu}$ and $\mathrm{Wu}, 2001,2005)$ and as backpropagators for seismic wave imaging/migration in both acoustic and elastic media (e.g., Wu and Xie, 1994; Huang and Wu, 1996; Huang et al., 1999a, 1999b; Jin and Wu, 1999; Jin et al., 1999; Xie and Wu, 1998, 2005; Xie et al., 2000).

The rest of this chapter is divided into three sections. We first briefly describe the basic concept of the one-way propagator for simulating elastic wave propagation in crustal wave guide. In the second part, we give a systematic review of the screen propagator for the $\mathrm{SH}$ wave propagation in complex crustal waveguides including irregular topography. In the last part, we present the P-SV Lg screen propagator. 


\section{A Brief Description of the Generalized Screen Propagator FOR GUIDED WAVES}

In the crustal wave guide environment, major part of wave energy is carried by forward propagating waves, including forward scattered waves. The Lg energy, which is in the form of guided waves, is carried by forward propagating waves bouncing up and down between the free surface and major geophysical discontinuities such as the Moho and Conrad discontinuities. Beyond the critical reflection angle, these waves are systematically dominated by small-angle waves (relative to the main propagation direction) trapped in the crustal waveguides. Therefore, neglecting backscattered waves in the propagation during numerical modeling will not modify the main features of regional waves in most cases. With this approximation, the modeling method becomes a forward marching algorithm in which the next step of propagation depends only on the present values of the wavefield in a transverse cross-section and the heterogeneities between the present cross-section and the next one (within an extrapolation interval). To formulate the problem, we divide the crustal wave guide into a sequence of vertical slabs. The horizontal direction is chosen as the main propagation direction. The geometry of the model is shown in Fig. 2a. Choosing one slab as the example, Fig. 2b shows the interaction between the incident waves and the slab. By introducing the local Born approximation, both wavefields and the elastic parameters can be separated into two parts, the background values and the perturbations. The "thin-slab" must be thin enough to satisfy the local Born approximation: the scattered field due to the heterogeneities in the slab be much smaller than the incident field. The incident $\mathrm{P}$ - and $\mathrm{S}$-waves $\mathbf{u}_{0}^{P}$ and $\mathbf{u}_{0}^{S}$ enter the slab from the vertical plane at $x_{0}$. After the incident waves pass through the thin-slab between $x_{0}$ and $x_{1}$, and interacting with the heterogeneities within it, there will be both incident

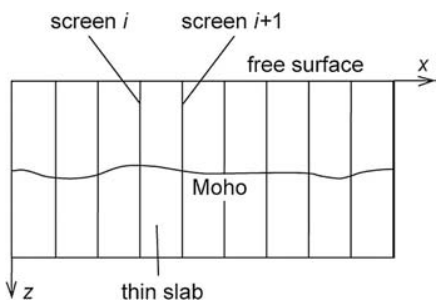

(a)

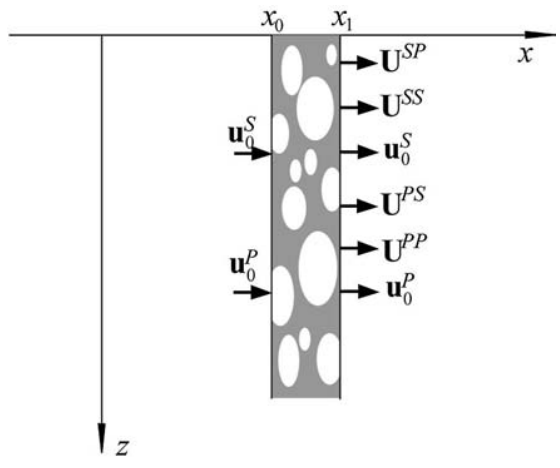

(b)

FIG. 2. (a) Geometry using screen method to simulate Lg wave; (b) Sketch showing the interaction between the incident waves and a thin slab. 
waves and different types of forward scattered waves at the exit plane at $x_{1}$. The new P-wave $\mathbf{u}^{P}=\mathbf{u}_{0}^{P}+\mathbf{U}^{P P}+\mathbf{U}^{S P}$ is composed of incident P-wave and scattered P-waves $\mathbf{U}^{P P}$ and $\mathbf{U}^{S P}$, respectively from incident $\mathrm{P}$ - and $\mathrm{S}$-waves, and the new S-wave $\mathbf{u}^{S}=\mathbf{u}_{0}^{S}+\mathbf{U}^{P S}+\mathbf{U}^{S S}$ is composed of incident S-wave and scattered S-waves $\mathbf{U}^{P S}$ and $\mathbf{U}^{S S}$, respectively. The propagation and scattering in the thin-slab can be formulated using the perturbation theory and calculated by two separated steps: (1) The interactions between the incoming waves and the heterogeneities are conducted in the spatial domain, accounting for the scattering and the coupling between different wave types. (2) Plane wave propagation through the background medium is conducted in the wavenumber domain by simple phaseshift. In both domains, the calculations are local and highly efficient. There is no time-consuming spatial or wavenumber domain convolution involved. Forward and inverse fast Fourier transforms (FFT) shuttle the wavefield between the two domains. By iteratively using this process and making the output from one slab as the input of the next slab, the wavefield can be propagated through the entire model.

The conventional wavenumber integral method is for the horizontally layered model and the integral is along horizontal wavenumber $k_{x}$. By contrast, the elastic screen method for propagating guided waves in crustal environment uses vertical screens and the wavenumber integration is carried out along the vertical $k_{z}$ axis. Under this geometry, the postcritical reflections become small-angle events with respect to the main propagation direction (the $x$-direction), therefore, the generalized screen propagator (GSP) methods based on small angle approximation is suitable for modeling $\mathrm{Lg}$ waves. Our discretized model is composed of vertical thin-slabs and therefore the wavenumber integral is along vertical wavenumber $k_{z}$, resulting in different features compared with the traditional wavenumber integration. Figure 3 is a sketch showing the difference between two integral axes. For the reflected $\mathrm{P}$ - and $\mathrm{S}$-waves coupled at a horizontal free surface, both waves have the same horizontal wavenumber (Fig. 3a). Choosing the horizontal wavenumber as the integral variable, $\mathrm{P}$ - and $\mathrm{S}$-waves have the same sampling points in the $k_{x}$ axis. The Rayleigh pole, which contributes to the generation of Rayleigh wave, has a unique location on the $k_{x}$ axis (Figs. 3a and b). However, choosing the vertical wavenumber $k_{z}$ as the integral variable, the $\mathrm{P}$ - and $\mathrm{S}$-waves have different sampling points. The Rayleigh pole in the real $k_{x}$ axis splits into two points in the imaginary $k_{z}$ axis (Fig. 3c). This makes resampling necessary when calculating converted waves. Fast Fourier transform with regular sampling interval cannot be used in the case of vertical wavenumber integration for converted waves. All these introduce additional complexity in wavenumber integration and special treatment is required. However, the general principle of plane wave (including the inhomogeneous plane waves) superposition for representing point sources still holds.

GSP is accurate for small-angle propagation and scattering (near horizontal for the crustal waveguide environment). A half-space screen propagator has 
(a)
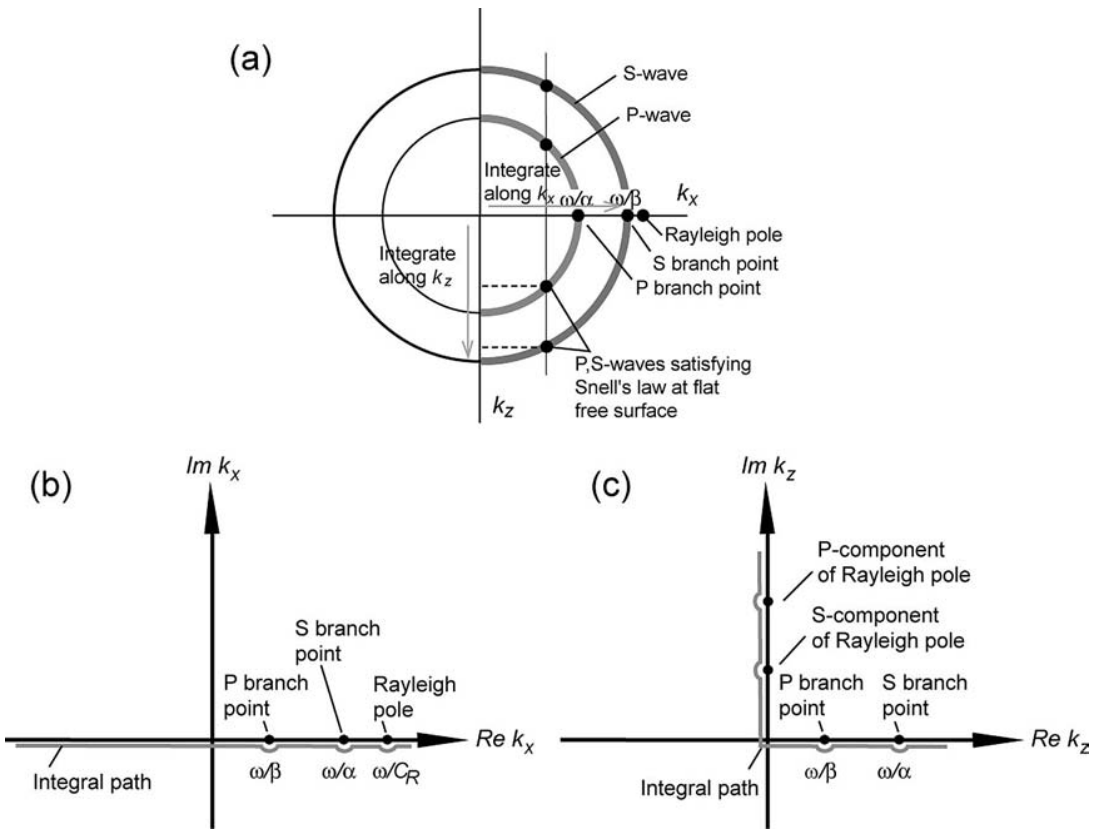

FIG. 3. Sketch showing different wavenumber domain integrals: (a) Dispersion relation in the $k_{x}-k_{z}$ plane; (b) Traditional wavenumber integration in the complex $k_{x}$ plane; (c) Wavenumber integration in the complex $k_{z}$ plane for the generalized screen method using vertical screens in a half-space.

been introduced by Wu et al. (1996, 1997, 1998, 2000a, 2000b) to accommodate the free-surface boundary condition and treat the $\mathrm{SH}$ wave propagation in complex crustal waveguides. The new one-way method for modeling regional SH waves has been calibrated extensively with various full-wave methods for different crustal models, such as the wavenumber integration method for flat structures and full-wave finite-difference method for heterogeneous crustal waveguides. Excellent agreement with these methods demonstrated the validity and accuracy of the new one-way method. For a model with propagation distance of $250 \mathrm{~km}$, dominant frequency at $0.5 \mathrm{~Hz}$ and with similar accuracy, the GSP method is about 300 times faster than the finite-difference method. The GSP method has been applied to the simulation of Lg propagation in random media for calculating the related energy partition and attenuation (Wu et al., 2000b). It is found that the leakage attenuation of $\mathrm{Lg}$ waves caused by forward large-angle scattering from random heterogeneities, which scatters the guided waves out of the trapped modes resulting in energy leaking into the mantle, may contribute significantly to $\mathrm{Lg}$ attenuation and blockage in some regions. The apparent $Q$ for leakage attenuation, as a function of normalized scale length 
$k a$ of the random heterogeneities, agrees well with the scattering theory. Later, the SH screen propagator is extended to the case of irregular surface topography by conformal or non-conformal topographic transforms (Wu et al., 1999; $\mathrm{Wu}$ and $\mathrm{Wu}, 2001$ ). In the conformal transform method, the coordinate system is rotated according to the local topographic slope, and the mirror image method can be applied to the local plane surface; the non-conformal method is a surface flattening transform which turns the free surface topography into modified volume perturbations of elastic parameters. The former method is suitable to deal with smoothly varying topography, while the latter method can treat rough but moderate topography.

In the P-SV wave case, the derivation and application of one-way GSP screen propagators are much more complicated. Unlike for SH waves, the mirror image method of generating the half-space Green's function cannot be used to account for the effect of the free surface. Plane wave reflection calculations are incorporated into the elastic screen method (Wu et al., 2000c). Body waves, including the reflected and converted waves, can be calculated by real wavenumber integration; while surface waves (Rayleigh waves) can be obtained with imaginary wavenumber integration. Numerical tests show good agreement with the theory. More work has to be conducted for the coupling among the body waves, guided wave and surface wave caused by lateral heterogeneities and irregular topography.

\section{SH WAVE CASE}

\subsection{Half-Space Screen Propagator}

For a 2-D SH problem, only the $y$-component of the displacement field, noted as $u$, exists. With the perturbation method, the medium and the wave field are decomposed into

$$
\begin{aligned}
& \rho=\rho_{0}+\delta \rho, \\
& \mu=\mu_{0}+\delta \mu, \\
& u=u^{0}+U,
\end{aligned}
$$

where $\rho_{0}$ and $\mu_{0}$ are the density and shear rigidity of the background medium, $\delta \rho$ and $\delta \mu$ are their corresponding perturbations, $u^{0}$ is the primary field and $U$ is the scattered field. The SH wave equation in the frequency domain can be written as

$$
\mu_{0} \nabla^{2} u+\omega^{2} \rho_{0} u=-\left\lfloor\omega^{2} \delta \rho u+\nabla \cdot \delta \mu \nabla u\right\rfloor,
$$

where $\nabla$ is the 2-D gradient operator and "." stands for inner product. Equation (1) is a scalar Helmholtz equation. With a half-space scalar Green's function $g^{h}$, the 
scattered field $U$ can be written as

$$
U\left(\mathbf{r}_{1}\right)=k^{2} \int_{v} \mathrm{~d}^{2} r\left\{g^{h}\left(\mathbf{r}_{1} ; \mathbf{r}\right) \varepsilon_{\rho}(\mathbf{r}) u(\mathbf{r})-\frac{1}{k^{2}} \nabla g^{h}\left(\mathbf{r}_{1} ; \mathbf{r}\right) \cdot \varepsilon_{\mu}(\mathbf{r}) \nabla u(\mathbf{r})\right\} .
$$

Under the forward-scattering approximation, or more generally the multipleforescattering-single-backscattering (MFSB) (De Wolf, 1971, 1985; Wu and Huang, 1995; Wu, 1996), the total field and Green's function under the integration in the above equation can be replaced by their forward-scattering approximated counterparts, and the field can be calculated by a one-way marching algorithm along the $x$-direction using a dual domain technique (see Chapter 5 of this book). Note that the half-space Green's function must be used here to account for the free surface effect.

For each step of the marching algorithm under the forward-scattering approximation, the total field at $x_{1}$ is calculated as the sum of the primary field which is the field propagating in the half-space from $x^{\prime}$ to $x_{1}$, and the scattered field caused by the heterogeneities in the thin-slab between $x^{\prime}$ and $x_{1}$. The thickness of the slab should be made thin enough to ensure the validity of the local Born approximation. The Green's function in the homogeneous half-space can be obtained by the mirror image method. The stress should vanish at the free surface $z=0$. Therefore we have

$$
g_{0}^{h}\left(\mathbf{r}_{1} ; \mathbf{r}\right)=g_{0}\left(\mathbf{r}_{1} ; \mathbf{r}\right)+g_{0}\left(\mathbf{r}_{1} ; \mathbf{r}^{*}\right),
$$

where $g_{0}$ is the infinite homogeneous Green's function and $\mathbf{r}^{*}$ is the mirror image point of $\mathbf{r}$ with respect to the free surface.

The free space Green's function in wavenumber domain is (Wu, 1996)

$$
g_{0}\left(x_{1}, K_{z} ; x, z\right)=\frac{1}{2 \gamma} e^{i \gamma\left|x_{1}-x\right|} e^{-i K_{z} z}
$$

with

$$
\gamma=\sqrt{k^{2}-K_{z}^{2}}
$$

Therefore,

$$
g_{0}^{h}\left(x_{1}, K_{z} ; x, z\right)=\frac{1}{2 \gamma} e^{i \gamma\left(x_{1}-x\right)} 2 \cos \left(K_{z} z\right) .
$$

In a similar way we can obtain

$$
\nabla g_{0}^{h}=\frac{1}{2} e^{i \gamma\left(x_{1}-x\right)}\left\{\hat{e}_{x} 2 \cos \left(K_{z} z\right)-\hat{e}_{z}\left(K_{z} / \gamma\right) 2 i \sin \left(K_{z} z\right)\right\},
$$

where $\hat{e}_{x}$ and $\hat{e}_{z}$ are the unit vectors in the $x$-and $z$-directions, respectively.

Taking Fourier transform of Eq. (2) along $z_{1}$ for the case of a thin-slab perpendicular to the $x$-direction, and substitute the half-space Green's functions into it, the scattered field by the thin-slab can be calculated by (for details see Wu et al., 
2000a)

$$
\begin{aligned}
U\left(x_{1}, K_{z}\right)= & U_{\rho}\left(x_{1}, K_{z}\right)+U_{\mu}\left(x_{1}, K_{z}\right), \\
U_{\rho}\left(x_{1}, K_{z}\right)= & i k \int_{x^{\prime}}^{x_{1}} \mathrm{~d} x e^{i \gamma\left(x_{1}-x\right)} C\left[\frac{k}{\gamma} \varepsilon_{\rho}(z) u_{0}(z)\right], \\
U_{\mu}\left(x_{1}, K_{z}\right)= & i k \int_{x^{\prime}}^{x_{1}} \mathrm{~d} x e^{i \gamma\left(x_{1}-x\right)} \\
& \times\left\{C\left[\varepsilon_{\mu}(z) \bar{\partial}_{x} u_{0}(z)\right]-i S\left[\frac{K_{z}}{\gamma} \varepsilon_{\mu}(z) \bar{\partial}_{z} u_{0}(z)\right]\right\},
\end{aligned}
$$

where

$$
\varepsilon_{\rho}(\mathbf{r})=\frac{\delta \rho(\mathbf{r})}{\rho_{0}}, \quad \varepsilon_{\mu}(\mathbf{r})=\frac{\delta \mu(\mathbf{r})}{\mu_{0}},
$$

and $\gamma=\sqrt{k^{2}-K_{z}^{2}}$ is the propagating wavenumber in the $x$-direction, $K_{z}$ is transverse wavenumber along the $z$-axis, and

$$
\bar{\partial}_{x}=\frac{1}{i k} \frac{\partial}{\partial x}
$$

are dimensionless partial derivatives. In the above equations, $C[f(z)]$ and $S[f(z)]$ are the cosine and sine transforms:

$$
\begin{aligned}
& C[f(z)]=\int_{0}^{\infty} \mathrm{d} z 2 \cos \left(K_{z} z\right) f(z), \\
& S[f(z)]=\int_{0}^{\infty} \mathrm{d} z 2 \sin \left(K_{z} z\right) f(z),
\end{aligned}
$$

and $u_{0}, \bar{\partial}_{x} u_{0}$ and $\bar{\partial}_{z} u_{0}$ can be calculated by

$$
\begin{gathered}
u_{0}(x, z)=\frac{1}{2 \pi} \int_{-\infty}^{\infty} \mathrm{d} K_{z}^{\prime} e^{i K_{z}^{\prime} z} e^{i \gamma^{\prime}\left(x-x^{\prime}\right)} u_{0\left(x^{\prime}, K_{z}^{\prime}\right)} \\
=C^{-1}\left[e^{i \gamma^{\prime}\left(x-x^{\prime}\right)} u_{0}\left(x^{\prime}, K_{z}^{\prime}\right)\right] \\
\bar{\partial}_{x} u_{0}(x, z)=C^{-1}\left[e^{i \gamma^{\prime}\left(x-x^{\prime}\right)} \frac{\gamma^{\prime}}{k} u_{0}\left(x^{\prime}, K_{z}^{\prime}\right)\right], \\
\bar{\partial}_{z} u_{0}(x, z)=i S^{-1}\left[e^{i \gamma^{\prime}\left(x-x^{\prime}\right)} \frac{K_{z}^{\prime}}{k} u_{0}\left(x^{\prime}, K_{z}^{\prime}\right)\right] .
\end{gathered}
$$

The above equations are the general wide-angle formulation. When the energy of crustal guided waves is carried mainly by small-angle waves (with respect to the horizontal direction), the phase-screen approximation can be invoked to simplify the theory and calculations. Summing up the primary and scattered fields 
and invoking the Rytov transform results in the dual-domain expression of phasescreen propagator

$$
u\left(x_{1}, K_{z}\right) \approx e^{i \gamma\left(x_{1}-x^{\prime}\right)} C\left[e^{i k S_{s}(z)} u_{0\left(x^{\prime}, z\right)}\right],
$$

where $e^{i k S_{s}(z)}$ is the phase delay operator with

$$
S_{S}(z)=\frac{1}{2} \int_{x^{\prime}}^{x_{1}} \mathrm{~d} x\left[\varepsilon_{\rho}(x, z)-\varepsilon_{\mu}(x, z)\right] \approx \Delta x \bar{\varepsilon}_{s}(z),
$$

where $\bar{\varepsilon}_{S}(z)$ is the average $\mathrm{S}$-wave slowness perturbation over the thin-slab at depth $z$,

$$
\bar{\varepsilon}_{s}(z)=\frac{1}{x_{1}-x^{\prime}} \int_{x^{\prime}}^{x_{1}} \mathrm{~d} x \frac{s(x, z)-s_{0}}{s_{0}},
$$

with $s(x, z)=1 / v(x, z)$ and $\Delta x=\left(x_{1}-x^{\prime}\right)$ is the thin-slab thickness. Equation (12) is the SH phase-screen propagator for the half space. It has a similar form as the whole space propagator with the Fourier transform replaced by a cosine transform.

The phase-screen propagator has long been used in ocean acoustics to simulate long range acoustic wave propagation in the heterogeneous ocean due to internal waves. Most work in the literature deals with the stochastic treatment of waves in random media. For an introduction and brief summary of the work in that field, the reader is referred to Flatté et al. (1979). However, in this work we will use the half-space screen propagator for deterministic modeling in heterogeneous crustal waveguides.

\subsection{Treatment of the Moho Discontinuity}

The Moho discontinuity can be treated in two ways. One way is to put the impedance boundary conditions in the formulation, the other is to treat the parameter changes as perturbations and therefore incorporate the discontinuity into the screen interaction. In this paper, we adopt the latter approach because of its flexibility in treating irregular Moho discontinuity. The validity of the perturbation approach for the Moho discontinuity is verified by the comparison with wavenumber integration and full-wave finite-difference algorithms. Since for guided waves, or crustal waves with critical or post-critical reflections, the related mantle waves are nearly horizontal, the screen approximation is quite accurate in this case. The excellent agreements of the method with the wavenumber integration for flat Moho, and with the finite-difference method for irregular Moho demonstrate the validity of this approach (Wu et al., 2000a).

Figure 4 compares the reflection coefficients of the Moho discontinuity calculated using the theoretical equation (dotted line) and using phase-screen method (solid line). A constant velocity crust model $\left(v_{c}=3.5 \mathrm{~km} / \mathrm{s}, \rho_{c}=2.8 \mathrm{~g} / \mathrm{cm}^{3}\right.$, 


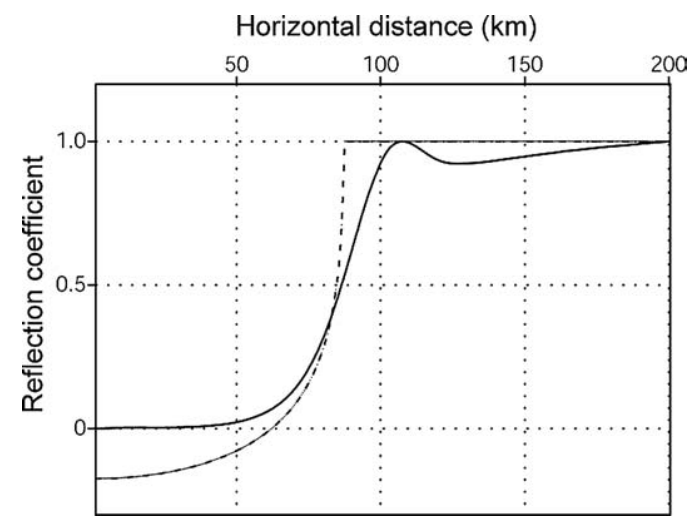

FIG. 4. Comparison of reflection coefficients at the Moho discontinuity. Dotted line denotes result from theoretical equation and solid line denotes result calculated using screen method. A constant velocity crust model is used in the calculation and the source is located $30 \mathrm{~km}$ above the Moho discontinuity.

$\left.v_{m}=4.5 \mathrm{~km} / \mathrm{s}, \rho_{m}=3.1 \mathrm{~g} / \mathrm{cm}^{3}\right)$ is used in the calculation and the source is located $30 \mathrm{~km}$ above the Moho. The curve from the screen method is obtained by using the root-mean-square (RMS) of the reflected waveforms. We see that the process of critical reflection is well matched, except that the transition from precritical to critical calculated by the screen method is not as sharp as the theoretical curve. This may be caused by the small phase error in the phase-screen approximation. In addition, the reflection for wide-angle incidence, especially for nearly vertical incidence, is not well modeled by the phase-screen method. This error results from the small angle approximation used in the screen formulation. However, this limitation occurs only at short range, well before the critical distance (around $80 \mathrm{~km}$ in this case). As can be verified using numerical simulations, the screen method behaves exceptionally well beyond the critical distance, making it a good candidate for guided wave simulation.

\subsection{Numerical Verifications and Simulation Examples}

In this section we show some examples demonstrating the validity of the method and its potential applications to various problems of regional wave propagation. First, we show a comparison between the screen method and a full-wave finite-difference method for a heterogeneous crustal model. Shown in Fig. 5a is a wave guide model with a crust necking. Figure $5 \mathrm{~b}$ shows the synthetic seismograms along a vertical profile at an epicentral distance of $250 \mathrm{~km}$. The thin lines are from the finite-difference method and the thick lines are from the screen method. The source is located at a depth of $2 \mathrm{~km}$ and the source time function has a dominant frequency of $0.5 \mathrm{~Hz}$. Figure 5 demonstrates excellent agreement 


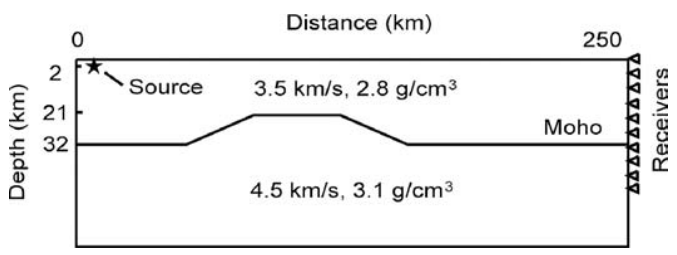

(a)

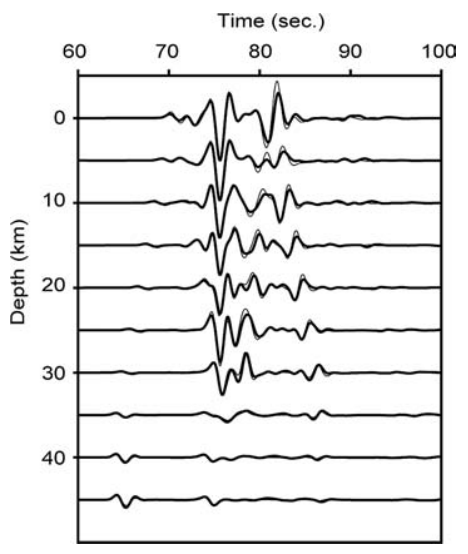

(b)

FIG. 5. Comparison of synthetic seismograms along a vertical profile at a distance of $250 \mathrm{~km}$. Shown in (a) is the velocity model with a laterally varying crustal wave guide. Shown in (b) are synthetic seismograms calculated using the screen method (thick lines) and a finite-difference method (thin lines). The source depth is $2 \mathrm{~km}$ and the source time function is a Gaussian derivative with a dominant frequency of $0.5 \mathrm{~Hz}$.

between the two methods. For this example, the GSP method is about 300 times faster than the finite-difference method. Note that the grid spacing used in the FD simulation was 3-4 times smaller than the stability requirement in order to reduce the numerical dispersion. Other comparisons with wavenumber integration and finite-difference methods can be found in Wu et al. (2000a, 2000b).

The importance of small-scale random heterogeneities to seismic wave propagation is well known. There are extensive publications on this subject in seismology. However, due to the complexity of the problem, the role of random heterogeneities in Lg excitation, propagation, attenuation and blockage is still unclear. For waves in complex crustal waveguides with random heterogeneities, there are still no analytical tools available for performing realistic calculations. Numerical simulation is a useful alternative to the theory. Some finite-difference simulations have been conducted (e.g., Frankel and Clayton, 1986; Frankel, 1989; Xie and Lay, 1994; Jih, 1996). Due to the limit of the computing power, the wavepropagation distances in the finite-difference simulations are relatively short. 

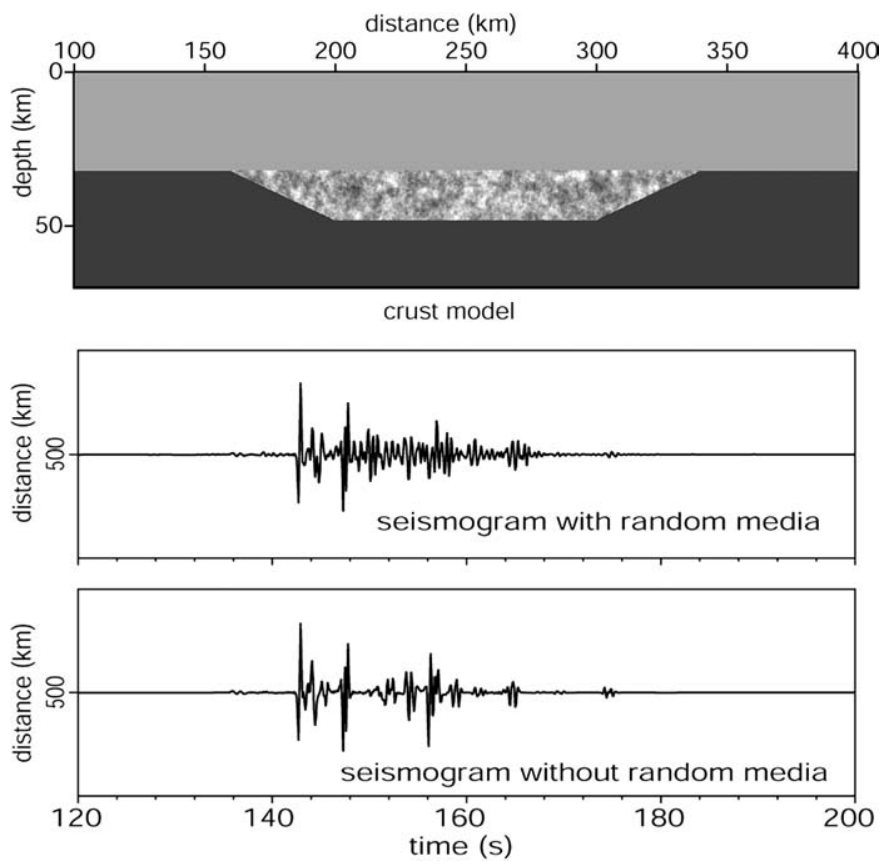

FIG. 6. A heterogeneous crustal model representing a mountain root with small-scale random heterogeneities (top panel). The comparisons between synthetic seismograms with and without random heterogeneities are shown on the middle and bottom panels, respectively.

Liu and Wu (1994) have done some numerical simulations using the phase-screen method, but the models simulated are limited to unbounded media. The development of the half-space GSP method enables us to simulate long distances, high-frequency wave propagation in complex crustal waveguides. We present here two numerical examples to demonstrate the capability of the GSP method.

Figure 6 shows a heterogeneous crustal model representing a "mountain root" with small-scale random heterogeneities. The top panel is the velocity model, and the comparisons between synthetic seismograms with and without random heterogeneities are shown on the middle and bottom panels, respectively. The heterogeneities have an exponential correlation function, with the scale length $a_{x}=$ $a_{z}=1.6 \mathrm{~km}$ (in horizontal and vertical directions, respectively). The RMS velocity perturbation is $5 \%$. The dominant frequency of the source time function is $2 \mathrm{~Hz}$. Figures $7 \mathrm{a}$ and $\mathrm{b}$ show a comparison of wavefield snapshots between models with and without random heterogeneities. We see that random heterogeneities drastically increase the complexity of the wavefield and the energy leakage to the upper mantle. 


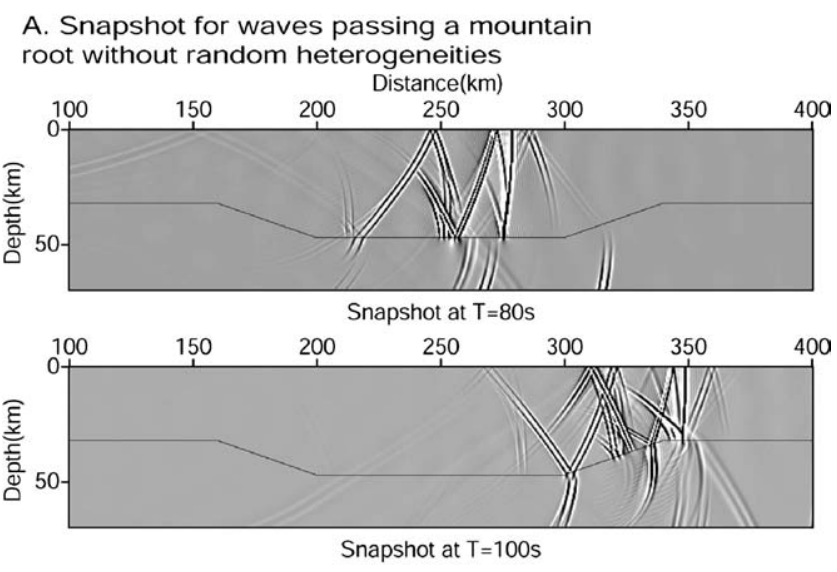

B. Snapshot for waves passing a mountain root with random heterogeneities

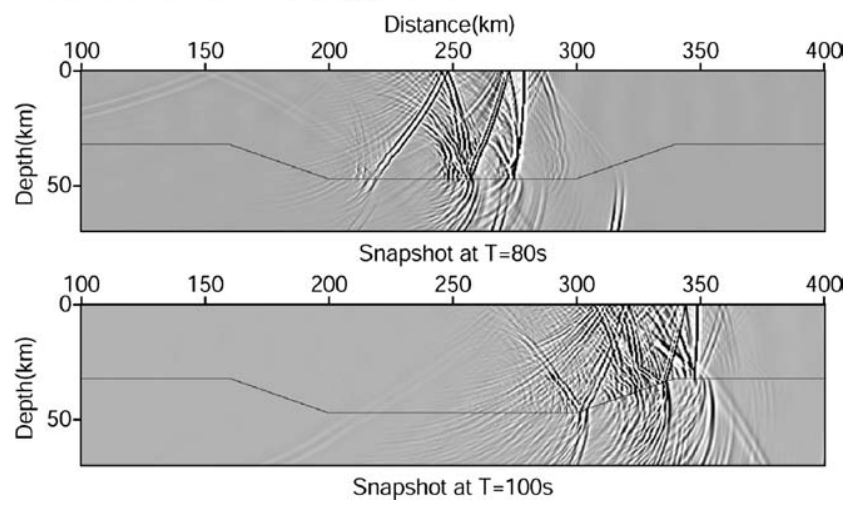

FIG. 7. Comparison between snapshots for waves passing through a "mountain root" with or without random heterogeneities, shown on A and B, respectively.

\subsection{Application to Energy Partition and Attenuation in Crustal Waveguide with Random Heterogeneities}

In heterogeneous crustal wave guides, the upper boundary is the free surface, which is a perfect reflector. The lower boundary of the wave guide is the Moho discontinuity. For waves incident on the Moho discontinuity, a part of the energy will leak into the upper mantle. However, for waves incident on the Moho with post-critical angles, total reflections occur and all the energy is reflected and trapped in the waveguide. Generally speaking, the guided wave energy can be expressed as

$$
E_{g}=C \int_{K_{z}<K_{c}}\left|u\left(K_{z}\right)\right|^{2} \mathrm{~d} K_{z},
$$


where $C$ is a constant, $K_{z}$ is the wavenumber in the $z$-direction, namely the transverse wavenumber, and $K_{c}$ is the critical wavenumber. Scattering processes can redistribute the energy in wavenumber domain, causing the leak of trapped energy into the upper mantle. In addition to the leakage loss, the guided waves suffer also the anelastic loss and backscattering loss. Assuming a homogeneous mantle and neglecting reverberation in the $x$-direction, the energy balance after propagating a short distance $\mathrm{d} x$ in the $x$-direction is

$$
E_{g}(x+\mathrm{d} x)=E_{g}(x)-E_{a}(x)-E_{b}(x)-E_{l}(x),
$$

where $E_{g}$ is the energy of guided crustal waves; $E_{a}$, energy lost due to absorption (anelastic loss); $E_{b}$, energy lost due to backscattering by random heterogeneities; $E_{l}$, energy lost due to leakage to the mantle caused by heterogeneities. In terms of different attenuation coefficients, it can be written as

$$
\frac{\mathrm{d} E_{g}}{\mathrm{~d} x}=-\left[\eta_{a}+\eta_{b}+\eta_{l}\right] E_{g}(x)=-\eta_{g} E_{g}(x),
$$

where $\eta_{a}=\left(E_{a} / E_{g}\right) / \mathrm{d} x, \eta_{b}=\left(E_{b} / E_{g}\right) / \mathrm{d} x$, and $\eta_{l}=\left(E_{l} / E_{g}\right) / \mathrm{d} x$ are the apparent attenuation coefficients for guided crustal waves. Equivalently,

$$
\eta_{g}=k Q_{g}^{-1}=k\left(Q_{a}^{-1}+Q_{b}^{-1}+Q_{l}^{-1}\right),
$$

where $Q$ 's are the corresponding apparent quality factors.

The leakage loss is the scattering loss due to the redistribution of $\mathrm{Lg}$ angular spectra. It is caused dominantly by large-angle forward scattering and therefore, it is several orders of magnitude larger than the backscattering loss, i.e. $\eta_{l} \gg \eta_{b}$. In the following, we will concentrate on the analysis of leakage loss of guided waves. For the leakage analysis, the angular spectrum representation or the energy distribution versus propagation angle (or vertical slowness) will be very useful and can show clearly which part of the energy would be trapped in the wave guide and which part of the energy would leak into the mantle.

In first-order approximation, the anelastic (intrinsic) attenuation is additive to the leakage loss, so that we can calculate and analyze these two attenuation mechanisms separately. For the Lg RMS amplitude attenuation, one more attenuation mechanism is involved:

$$
b_{g}=k Q_{g}^{-1}=k\left(Q_{a}^{-1}+Q_{b}^{-1}+Q_{l}^{-1}+Q_{d}^{-1}\right)
$$

where $Q_{d}^{-1}$ is the equivalent $Q$ of diffusion loss, which represents the amplitude decrease of $\mathrm{Lg}$ due to the transfer of coherent energy into incoherent energy $(\mathrm{Lg}$ coda) by random heterogeneities.

Shown in Fig. 8 is a comparison between angular spectra from a waveguide model with 5\% RMS random velocity perturbation in the crust and a reference flat crust without velocity perturbation. The perturbation has an exponential correlation function with horizontal and vertical characteristic scales (correlation lengths) of 5.0 and $3.0 \mathrm{~km}$, respectively. From the top panel to the bottom panel 

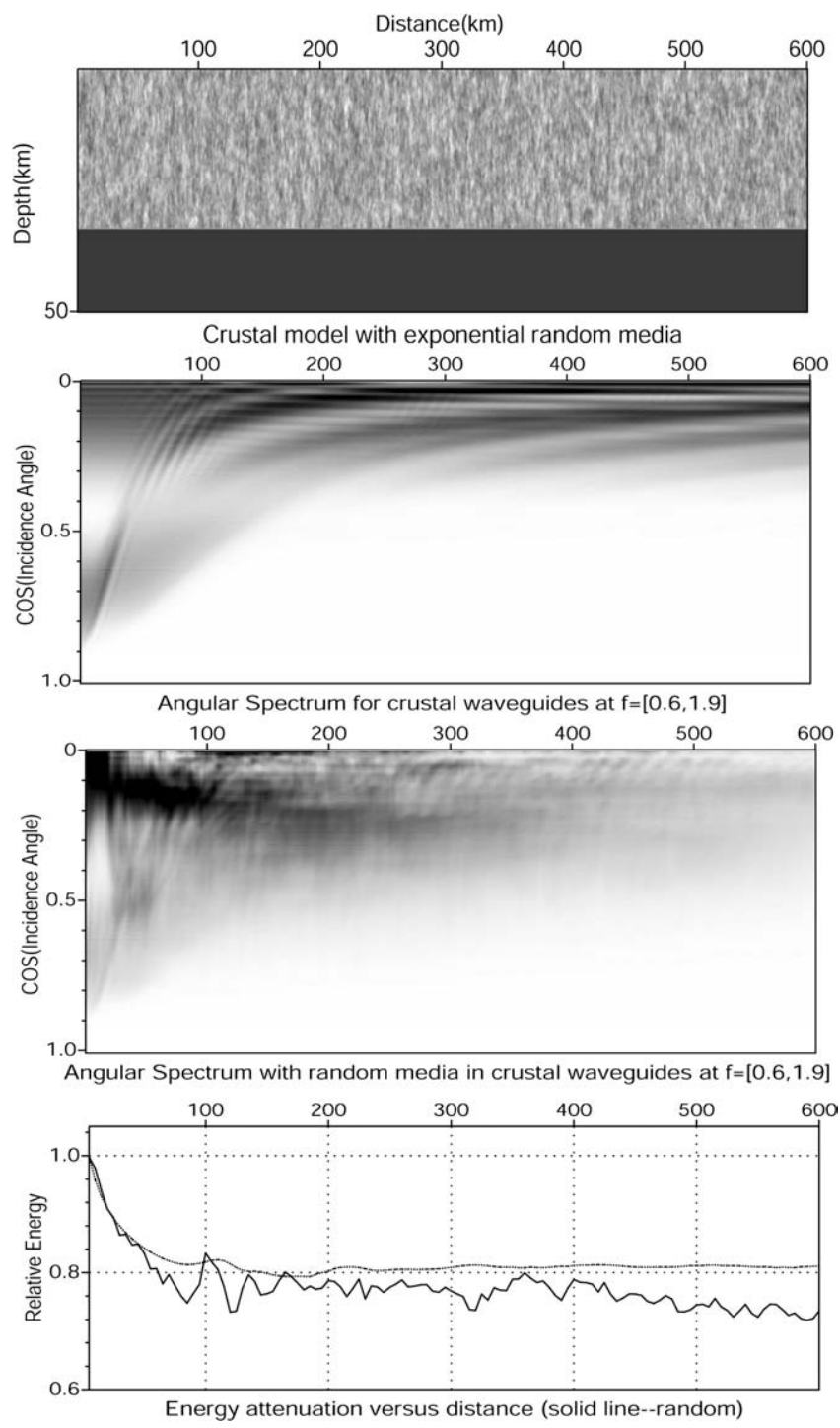

FIG. 8. Energy distribution for different crustal models. From top to bottom are: waveguide model with 5\% RMS velocity perturbations in the crust; energy angular spectra versus distance for a flat crust; energy angular spectra versus distance for random crust; and relative energy attenuations versus distance. The dotted line is for the flat crust model and the solid line is for the random crust model.

in Fig. 8, they are a random velocity model, energy distribution for homogeneous crust, energy distribution for random crust; and energy attenuation curves versus distances, respectively. For energy distributions, the vertical coordinate is the nor- 
malized vertical slowness $K_{z} / k$, corresponding to the cosine of incident angles (or sine of the grazing angles). Note that zero vertical slowness means horizontal propagation. The frequency range is between 0.6 and $1.9 \mathrm{~Hz}$. For the flat crust model, there is a considerable portion of energy with large vertical slowness (or steep angles) at the initial stage. After multiple reflections, energy with larger vertical slowness is depleted due to the leakage to the mantle, leaving the energy with small vertical slowness, i.e., the guided waves, propagating in the waveguide. For the model with random velocity perturbations, the distinct feature is the continuous energy repartition, moving from small (grazing) angle waves to large-angle waves due to scattering by small scale heterogeneities. The energy propagating with large angles tends to leak into the mantle and causes Lg-wave energy attenuation. The bottom panel of Fig. 8 is the wave energy attenuation versus the distance. The energy is calculated from synthetic seismograms on the free surface. The dotted line is for the reference (homogeneous) crust model. It can be seen that for this case, after passing $100 \mathrm{~km}$ or more, the energy is basically kept constant, which means that the trapped mode has been formed. The solid line is for the random waveguide. Due to the scattering, the energy is decreasing with distance.

Figure 9 gives the attenuation curves for different characteristic scales. The upper panel is the attenuation curve of total energy, which is the energy contained in the entire seismogram recorded on the surface. The thin solid line is for $k a=1$, the thick solid line is for $k a=10$, and the dashed line is for the reference (homogeneous) model. We see that for the reference model, the total energy remains constant beyond critical distance, which serves as a checking point for the numerical simulations. The middle panel gives the coherent $\mathrm{Lg}$ energy, which is calculated using waves within the Lg window (group velocity between $3.7 \mathrm{~km} / \mathrm{s}$ and $3.2 \mathrm{~km} / \mathrm{s}$ ) versus distance. Again, the thin, thick and dashed lines are for $k a=1, k a=10$ and the reference model, respectively. In both measurements, the cases with $k a=1$ are always associated with stronger attenuation than $k a=10$ cases. We also see that the coherent Lg energy corresponding to the peak amplitude suffers more attenuations than the total energy. This is due to the extra attenuation, i.e. the diffusion loss which scatters the waves out of the $\mathrm{Lg}$ window and transfers them into incoherent waves ( $\mathrm{Lg}$ coda). However, in these numerical simulations, there is no intrinsic attenuation, and leakage attenuation dominates. The difference between the coherent energy attenuation and the total energy attenuation is relatively small. In the bottom panel of Fig. 9, we plot the curve of apparent inverse quality factor for leakage attenuation $Q_{l}^{-1}$ versus the normalized scale length $(\mathrm{ka})$ of random heterogeneities, where $k=2 \pi / \lambda$ with $\lambda$ being the wavelength of the dominant frequency, and $a$ the correlation length. Since no intrinsic (anelastic) attenuation exists in the model and no backscattering is involved, the attenuation is solely caused by the leakage loss due to scattering. From the curve we see that $Q_{l}^{-1}$ reaches its peak at $k a \approx 1.5-2.0$ and keeps flat until $k a \approx 8.0$. This is a feature of large-angle 


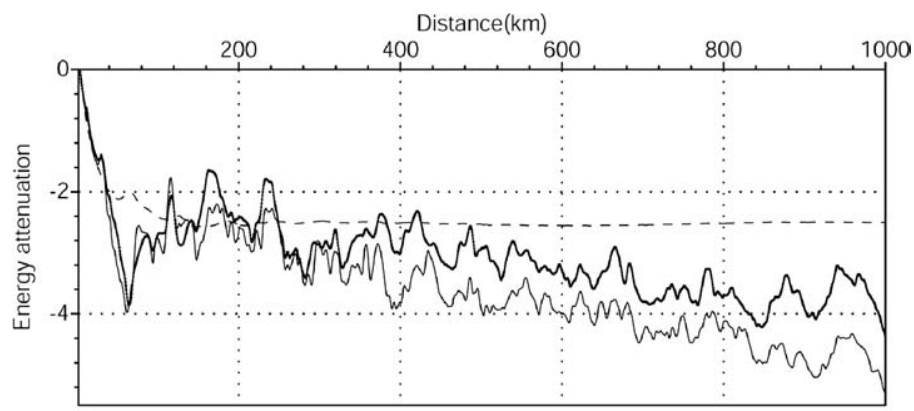

Energy attenuation versus ka (thin solid line - 1, thick - 10)

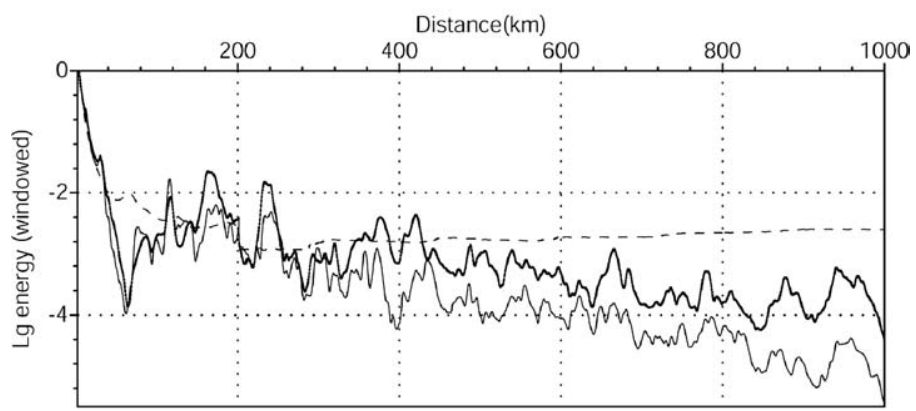

Lg energy attenuation versus ka (thin solid line - 1, thick - 10)

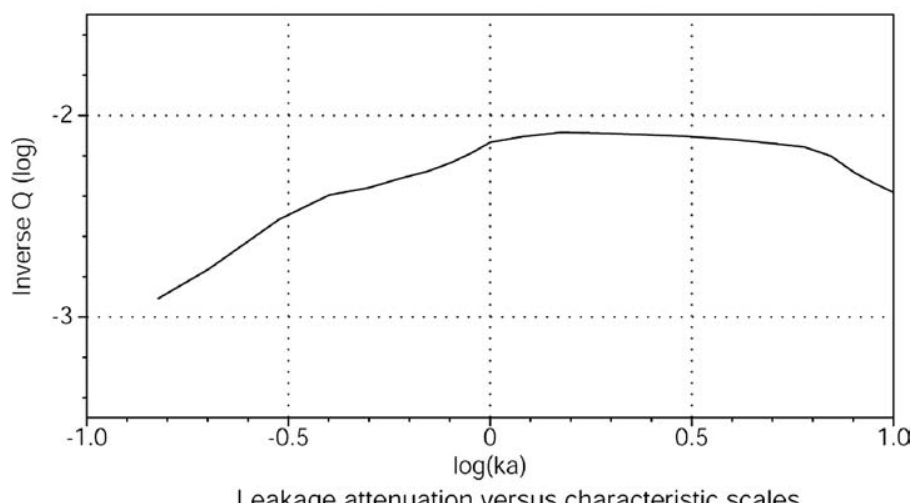

FIG. 9. Total energy attenuation (top panel), and windowed $L g$ energy attenuation between group velocities $3.1 \mathrm{~km} / \mathrm{s}$ and $3.7 \mathrm{~km} / \mathrm{s}$ (middle panel) versus distance for $k a=1$ (thin lines) and $\mathrm{ka}=10$ (thick lines). The bottom panel shows the equivalent $Q^{-1}$ for leakage attenuation versus the normalized scale length $\mathrm{ka}$. The dashed line is for the reference model of a homogeneous crust.

forward-scattering dominance. For backscattering, the maximum scattering $Q^{-1}$ is around $k a \approx 1.0$ and decreases rapidly at $k a>1.0$ for exponential correlation functions; while for large-angle forescattering, the plateau is quite wide after 
$k a=1.0(\mathrm{Wu}, 1982$; Frankel and Clayton, 1986). The numerical simulations agree well with the scattering theory. The values of the equivalent $Q$ (300-900 for $f_{0}=1 \mathrm{~Hz}$ ) are comparable with some observations (Xie and Mitchell, 1991; Xie, 1993). This suggests that the leakage attenuation caused by small-scale random heterogeneities may be responsible and even the dominant mechanism for some observed $\mathrm{Lg}$ attenuations and blockages.

\subsection{SH-Waves in Crustal Waveguides with Irregular Surface Topography}

Theoretical studies and observations show that surface topography is one of the important factors affecting $\mathrm{Lg}$ wave propagation. For example, irregular surface can cause anomalous variation of $\mathrm{Lg}$ amplitude along the propagation path (Sills, 1978; Geli et al., 1988; Bouchon and Barker, 1996). Methodologically, range-independent boundary conditions for flat surface must be replaced by range-dependent boundary condition for an irregular surface. In the case of surface topography, the global mirror symmetry no longer exists. To use the GSP method for solving range-dependent boundary condition problems, both conformal and non-conformal coordinate transforms were incorporated into the GSP method and their relative merits and accuracies were analyzed (Wu et al., 1999; $\mathrm{Wu}$ and $\mathrm{Wu}, 2001)$. The following is a summary of these two approaches.

\subsubsection{Conformal Coordinate Transform Method for Smoothly Varying Topography}

For a flat free surface, Wu et al. (2000a) derived a half-space GSP solution for $\mathrm{Lg}$ wave propagation. In the case of irregular topography, the global mirror symmetry for the problem no longer exists. However, taking a local plane-surface approximation for the topography, we can modify the mirror wavefield method to a local mirror wavefield method and apply the corresponding coordinate transform to obtain a GSP solution for the irregular topography.

Figure 10 shows the geometry of the derivation. Assume that $u_{0}^{+}\left(x^{\prime}, z\right)$ is the wavefield on the half-screen $S^{+}$in the lower half-space. To calculate the wavefield in the next screen with the existence of a locally dipping surface, we first obtain the mirror wavefield $u_{0}^{-}\left(\tilde{x}^{\prime}, \tilde{z}\right)$ on the half-screen $S^{-}$in the upper halfspace. The total wavefield in the next screen is composed of contributions from incident waves $u_{0}^{+}\left(x^{\prime}, z\right)$ and $u_{0}^{-}\left(\tilde{x}^{\prime}, \tilde{z}\right)$ plus the scattered field which is generated by the local heterogeneities in the thin-slab. The effects of the heterogeneities and the topography can be calculated separately for each step in the GSP method. The effect of the slant free-surface can be incorporated into the propagation integral. Assume $u_{t}(x, z)$ is the total field including the scattering effect of the volume heterogeneities. The wavefield $u\left(x_{1}, z_{1}\right)$ can be calculated by the Kirchhoff inte- 


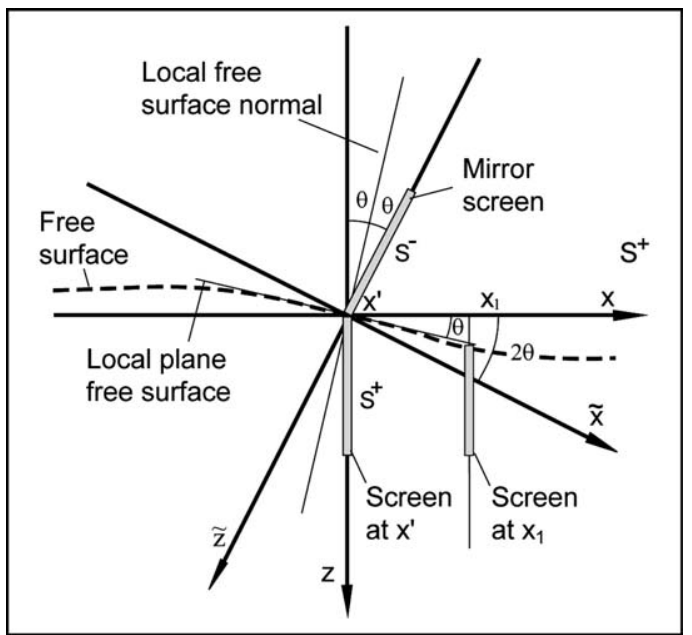

FIG. 10. Geometry of the conformal coordinate transform.

gral

$$
\begin{aligned}
u\left(x_{1}, z_{1}\right) & =\int_{S} \mathrm{~d} s\left\{g\left(x, z ; x_{1}, z_{1}\right) \frac{\partial u_{t}(x, z)}{\partial n}-\frac{\partial g\left(x, z ; x_{1}, z_{1}\right)}{\partial n} u_{t}(x, z)\right\} \\
& =\int_{S^{-}} \mathrm{d} s\{\cdots\}+\int_{S^{+}} \mathrm{d} s\{\cdots\},
\end{aligned}
$$

where $g(\cdot)$ is the Green's function for the full space with the background velocity, $S=S^{+}+S^{-}$is the integration surface composed of lower and upper halfsurfaces $S^{+}$and $S^{-}$, respectively. The Rayleigh integral can be used to replace the Kirchhoff integral for each half surface integral. For the lower half-surface the contribution of $S^{+}$is

$$
\begin{aligned}
u_{t}^{+}\left(x_{1}, z_{1}\right) & =-2 \int_{0}^{\infty} \mathrm{d} z u_{t}^{+}(x, z) \frac{\partial g\left(x, z ; x_{1}, z_{1}\right)}{\partial n} \\
& =\frac{1}{2 \pi} \int \mathrm{d} K_{T} e^{i K_{T} z_{1}} u_{t}^{+}\left(x_{1}, K_{T}\right),
\end{aligned}
$$

where

$$
u_{t}^{+}\left(x_{1}, K_{T}\right)=e^{i \gamma\left(x_{1}-x\right)} \int_{0}^{\infty} \mathrm{d} z_{1} u_{t}^{+}\left(x, z_{1}\right) e^{-i K_{T} z_{1}} .
$$

Here $u_{t}^{+}(x, z)$ is the total wavefield composed of incident field $u_{0}^{+}(x, z)$ and the scattered field $U^{+}(x, z)$ caused by the heterogeneities within the slab (see Wu, 1994; Wu et al., 2000a). If we put the slab entrance at $x=x^{\prime}$ and the wavefield on the entrance surface $S^{+}$as $u_{t}^{+}\left(x^{\prime}, z^{\prime}\right)$, then

$$
u_{t}^{+}\left(x^{\prime}, z^{\prime}\right)=u_{0}^{+}\left(x^{\prime}, z^{\prime}\right)+U^{+}\left(x^{\prime}, z^{\prime}\right) \text {, }
$$


where

$$
\begin{aligned}
U^{+}\left(x^{\prime}, z^{\prime}\right)= & k^{2} \int_{x^{\prime}}^{x_{1}} \mathrm{~d} x e^{-i \gamma\left(x_{1}-x^{\prime}\right)} \int_{0}^{\infty} \mathrm{d} z\left\{g\left(x, z ; x_{1}, z_{1}\right) \varepsilon_{\rho}(x, z) u_{0}(x, z)\right. \\
& \left.-\frac{1}{k^{2}} \nabla g\left(x, z ; x_{1}, z_{1}\right) \cdot \varepsilon_{\mu}(x, z) \nabla u_{0}(x, z)\right\} .
\end{aligned}
$$

For the bent upper half surface, we perform a coordinate transform by clockwise rotation of $2 \theta$ to a new coordinate system $(\tilde{x}, \tilde{z})$. Taking the downward direction as positive $z$-direction and the rotation angle from $x$ to $z$ as positive, the relation connecting the two systems is

$$
\begin{aligned}
& \tilde{x}=x \cos 2 \theta+z \sin 2 \theta, \\
& \tilde{z}=-x \sin 2 \theta+z \cos 2 \theta .
\end{aligned}
$$

In the new system, the surface $S^{-}$is parallel to the $\tilde{z}$-axis, so that

$$
u_{t}^{-}\left(\tilde{x}_{1}, \tilde{K}_{T}\right)=e^{i \tilde{\gamma}\left(\tilde{x}_{1}-\tilde{x}\right)} \int_{-\infty}^{0} \mathrm{~d} \tilde{z}^{\prime} u_{t}^{-}\left(\tilde{x}^{\prime}, \tilde{z}^{\prime}\right) e^{-i \tilde{K}_{T} \tilde{z}^{\prime}}
$$

where $u_{t}^{-}\left(\tilde{x}^{\prime},-\tilde{z}^{\prime}\right)=u_{t}^{+}\left(x^{\prime}, z^{\prime}\right)$. The field in the space domain can be obtained by synthesizing the contributions from all plane waves

$$
u_{t}^{-}\left(\tilde{x}_{1}, \tilde{z}\right)=\int \mathrm{d} \tilde{K}_{T} e^{i \tilde{\gamma}\left(\tilde{x}_{1}-\tilde{x}^{\prime}\right)} e^{i \tilde{K}_{T} \tilde{z}^{\prime}} u_{t}^{-}\left(\tilde{x}^{\prime}, \tilde{K}_{T}\right),
$$

where

$$
u_{t}^{-}\left(\tilde{x}^{\prime}, \tilde{K}_{T}\right)=\int_{-\infty}^{0} \mathrm{~d} \tilde{z}^{\prime} u_{t}^{-}\left(\tilde{x}^{\prime}, \tilde{z}^{\prime}\right) e^{-i \tilde{K}_{T} \tilde{z}^{\prime}}
$$

Transform back to the original coordinate system, resulting in

$$
\begin{aligned}
u_{t}^{-}\left(x_{1}, z_{1}\right)= & \int \mathrm{d} \tilde{K}_{T} \exp \left\{i \left[\left(\tilde{\gamma} \cos 2 \theta-\tilde{K}_{T} \sin 2 \theta\right)\left(x_{1}-x^{\prime}\right)\right.\right. \\
& \left.\left.+\left(\tilde{\gamma} \sin 2 \theta+\tilde{K}_{T} \cos 2 \theta\right) z_{1}\right]\right\} u_{t}^{-}\left(\tilde{x}^{\prime}, \tilde{K}_{T}\right) .
\end{aligned}
$$

We see that in the original coordinate system, the effective transversal and propagating wavenumbers are

$$
\begin{aligned}
& K_{T}=\tilde{\gamma} \sin 2 \theta+\tilde{K}_{T} \cos 2 \theta, \\
& \gamma=\tilde{\gamma} \cos 2 \theta-\tilde{K}_{T} \sin 2 \theta .
\end{aligned}
$$

If we transform the $\left(\tilde{K}_{T}, \tilde{\gamma}\right)$ system into $\left(K_{T}, \gamma\right)$,

$$
u_{t}^{-}\left(x_{1}, z_{1}\right)=\int \mathrm{d} K_{T} u_{t}^{-}\left(\tilde{x}^{\prime}, K_{T} \cos 2 \theta-\gamma \sin 2 \theta\right) e^{i \gamma\left(x_{1}-x^{\prime}\right)} e^{i K_{T} z_{1}} .
$$


The total field is a summation of the contributions from both $u_{t}^{+}\left(x_{1}, z_{1}\right)$ and $u_{t}^{-}\left(x_{1}, z_{1}\right)$

$$
\begin{aligned}
u\left(x_{1}, z_{1}\right)= & \int \mathrm{d} K_{T} e^{i \gamma\left(x_{1}-x^{\prime}\right)} e^{i K_{T} z_{1}} \\
& \times\left[u_{t}^{+}\left(x^{\prime}, K_{T}\right)+u_{t}^{-}\left(\tilde{x}^{\prime}, K_{T} \cos 2 \theta-\gamma \sin 2 \theta\right)\right] .
\end{aligned}
$$

When small-angle waves prevail such as in the case of Lg propagation, the spectral interpolation in Eq. (32) can be avoided and replaced by operations in the space domain using a narrow-angle approximation. From (23), it can be seen that to calculate the reflection response we need to find the spectral component $u_{t}^{+}\left(-\tilde{K}_{T}\right)$. We try to obtain the approximate space-domain operations corresponding to the wavenumber-domain interpolation. We know that

$$
u_{t}^{+}\left(-\tilde{K}_{T}\right)=\int_{0}^{\infty} \mathrm{d} z e^{i\left(-K_{T} \cos 2 \theta+\gamma \sin 2 \theta\right) z} u_{t}^{+}(z) .
$$

With narrow-angle approximation, $\gamma \approx k$, therefore,

$$
u_{t}^{+}\left(-\tilde{K}_{T}\right)=\int_{0}^{\infty} \mathrm{d} z^{\prime} e^{i K_{T} z^{\prime}}\left[\frac{1}{\cos 2 \theta} e^{i k(\tan 2 \theta) z^{\prime}} u_{t}^{+}\left(\frac{z^{\prime}}{\cos 2 \theta}\right)\right],
$$

where $\theta$ is the dipping angle of the free surface at $x=x^{\prime}$. We see that the wavenumber-domain interpolation is transformed into a space-domain operation which is a modulation plus a coordinate stretching. For a flat surface, Eq. (32) reduces to the original half-space GSP method (Wu et al., 2000a).

Shown in Fig. 11 are the synthetic seismograms obtained using the conformal screen method for a Gaussian hill model (Fig. 11a). The Gaussian hill is represented by $h(x)=-h_{0} \exp \left[-\left(x-x_{0}\right)^{2} / 2 \sigma^{2}\right]$ with $x_{0}=62.25 \mathrm{~km}, h_{0}=4 \mathrm{~km}$, and $\sigma=9.129 \mathrm{~km}$. Synthetic seismograms calculated with a more accurate boundary element method (Fu and $\mathrm{Wu}, 2001)$ are also given as a reference. The solid lines are from the screen method and the dashed lines are from boundary element method. The comparison indicates that the screen method gives a satisfactory result. It correctly modeled waveforms between distance 60 and $70 \mathrm{~km}$, where two reflections from the convex free surface interfere with each other and generate complex waveforms. Note that the coordinate stretch $z / \cos 2 \theta$ increases very fast with large angle $\theta$, the conformal screen method works only for smoothly varying topography.

\subsubsection{Non-Conformal Coordinate Transform Method for Rough Topography}

Another alternative approach is to incorporate surface flattening transform into the GSP method. The transform converts surface height perturbations into modified volume perturbations. In this way the range-dependent boundary condition becomes a stress release boundary condition on a flat surface in the new coordinate system where the half-space GSP method is applicable. The transform is 


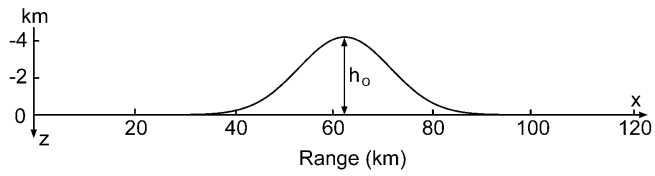

(a)

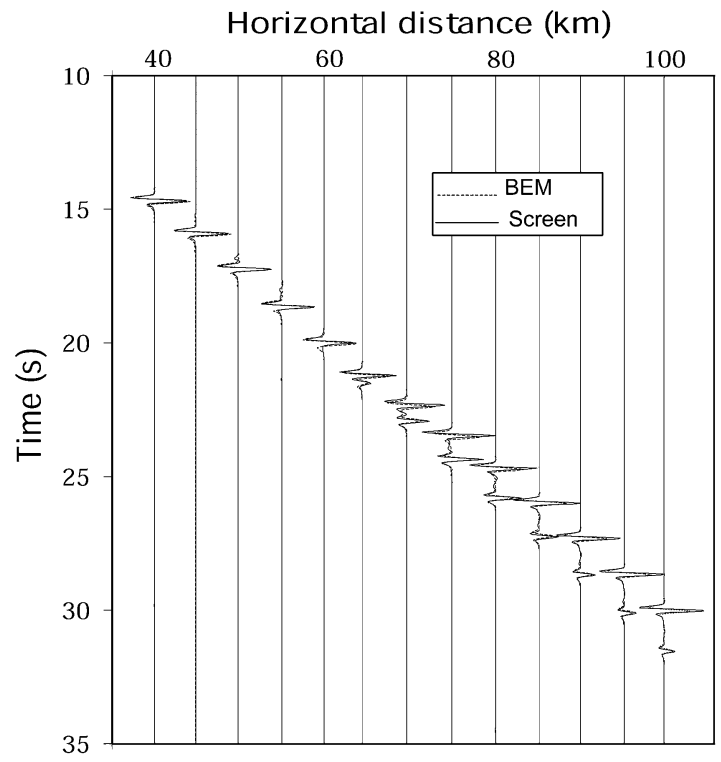

(b)

FIG. 11. (a) Velocity model with a Gaussian hill topography and (b) synthetic seismograms calculated from this model. For the calculation, $\mathrm{d} x=\mathrm{d} z=0.25 \mathrm{~km}$ and $\mathrm{d} t=0.05 \mathrm{~s}$. The source is located at a depth of $32 \mathrm{~km}$ and the dominant frequency of source time function is $3 \mathrm{~Hz}$. Receivers are on the free surface. The solid lines are synthetic seismograms calculated using the screen method with a conformal transform, and the dashed lines are synthetic seismograms calculated with boundary element method (Fu and $\mathrm{Wu}, 2001)$.

defined as (Beillis and Tappert, 1979)

$$
\left\{\begin{array}{l}
\chi=x, \\
\zeta=z-h(x),
\end{array}\right.
$$

where $h(x)$ is the height function of free surface. Equation (35) shows that the transform gives only a shift to depth variable $z$, i.e., depth measurement starts from the free surface. Thus, it is a non-conformal transform. Using the above transform and perturbation theory, the original half-space screen propagator becomes (Wu and $\mathrm{Wu}, 2001)$ 


$$
\begin{aligned}
\hat{u}\left(\chi_{1}, k_{\zeta}\right)= & e^{i \gamma \Delta \chi} C\left\{e^{i k_{0} S_{s}(\zeta)} C^{-1}\left[\hat{u}_{0}\left(\chi^{\prime}, k_{\zeta}^{\prime}\right)\right]\right. \\
& \left.-Z\left(\chi_{1}\right) \frac{\hat{\mu}(\zeta)}{\mu_{0}} S^{-1}\left[k_{\zeta}^{\prime} \hat{u}_{0}\left(\chi^{\prime}, k_{\zeta}^{\prime}\right)\right]\right\},
\end{aligned}
$$

where $C$ and $C^{-1}$ are the forward and inverse cosine transforms, and $S^{-1}$ is the inverse sine transform defined by Eq. (9), $\mu_{0}$ is shear modulus of background medium, $S_{S}$ is the relative slowness perturbation of the thin-slab and given by Eq. (13), and

$$
Z\left(\chi_{1}\right)=h\left(x_{1}\right)-h\left(x^{\prime}\right) .
$$

Equation (36) is expressed in the new coordinates $(\chi, \zeta)$. It is clear that the second term in the bracket in Eq. (36) comes from the roughness of topography, which is proportional to the height difference of the adjacent two screens for each forward step. For the upgoing slope, $Z(\chi)<0$, the field scattered by topography is in-phase with the background field and strengthens the background field, while for downgoing slope $Z(\chi)>0$, the field scattered by topography is out-phase with the background field and weakens the background field. Equation (36) is computationally efficient, in which all calculations can be done by FFT.

Shown in Fig. 12 are synthetic seismograms calculated using the nonconformal screen method for the Gaussian hill model shown in Fig. 11. The solid lines are from the screen method and the dashed lines are from the boundary element method. The excellent agreement between the two methods is clearly seen except at the vicinity of the hill top where a small discrepancy exists both in wave shapes and amplitudes. The error can be reduced by using a smaller step

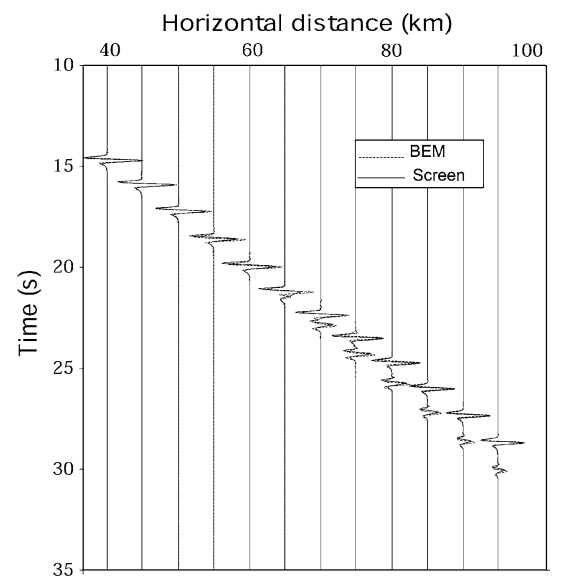

FIG. 12. Synthetic seismograms for a Gaussian hill model (Fig. 11a). The solid lines are calculated using the screen method with a non-conformal transform and the dashed lines are calculated using the boundary element method. The parameters for the calculation are the same as in Fig. 11. 


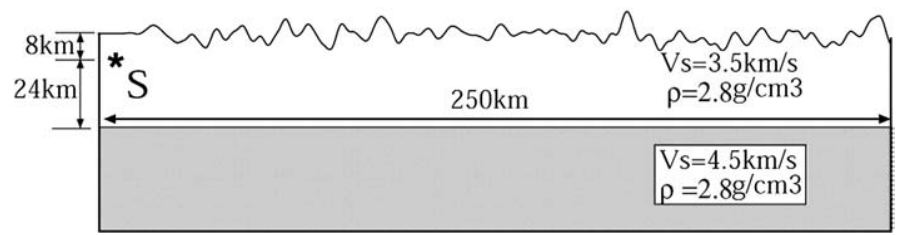

(a)

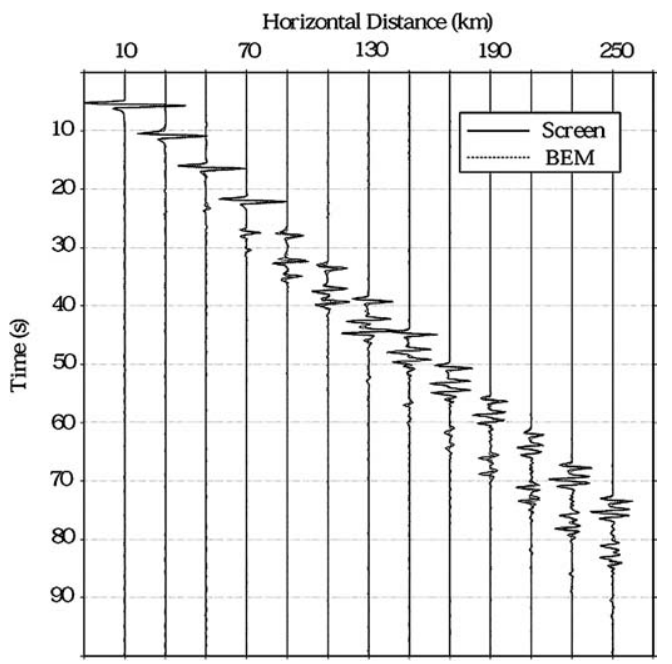

(b)

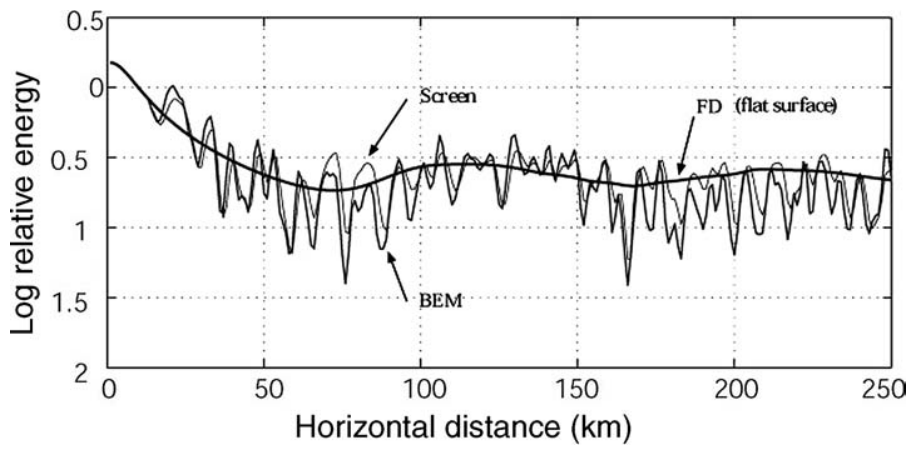

(c)

FIG. 13. (a) A crustal model with a rough random surface. The correlation length is $2.5 \mathrm{~km}$, RMS perturbation is $0.6 \mathrm{~km}$. (b) Synthetic seismograms, and (c) energy distribution versus horizontal distance. (b) and (c) show a comparison between the non-conformal screen method and BE method for a crustal waveguide with a rough random surface. The thick smoothly varying curve in (c) is calculated with finite difference method for a uniform waveguide. The source is located at the depth of $8 \mathrm{~km}$, the dominant frequency of the source time function is $1 \mathrm{~Hz}$. 
length $\Delta x$. For forward marching algorithms, the step length $\Delta x$ can be adjusted according to the roughness of topography. The more severe the topography is, the finer the step length $\Delta x$ should be. Therefore, the non-conformal screen method can handle more severe topography than the conformal screen method. Figure 13a shows a crustal model with a rough random surface used for testing feasibility and accuracy of the non-conformal screen method. The correlation length is $2.5 \mathrm{~km}$, RMS height fluctuation is $0.6 \mathrm{~km}$. Figures $13 \mathrm{~b}$ and $\mathrm{c}$ show a comparison of synthetic seismograms calculated by the non-conformal screen method and $\mathrm{BE}$ method, and the corresponding energy attenuation curves, respectively. The thick smoothly varying curve in Fig. 13c is the energy distribution for a similar waveguide but with a flat free surface. We see that the presence of a rough random surface makes the waveforms and attenuation curves more complicated. Except for large-angle Moho reflections, the results of the screen method agree well with those of the BE method. However, for this example, the screen method took about 35 minutes, while the BE method took about 72 hours.

Figure 14 shows an investigation of the combining effect of rough topography and volume heterogeneity on $\mathrm{Lg}$ wave propagation using the non-conformal screen method. The rough topography is the same as shown in Fig. 13. The heterogeneities are velocity variations only. The correlation lengths are $6 \mathrm{~km}$ in range and $4 \mathrm{~km}$ in depth, RMS velocity fluctuations are $5 \%$ and $10 \%$, respectively. The thickly dashed line calculated by finite-difference method for a uniform crustal waveguide is used as a reference. We see from Fig. 14 that random heterogeneities combined with rough topography drastically increase the attenuation of high frequency $\mathrm{Lg}$ waves. This example shows that the non-conformal screen method can handle the effects of both volume heterogeneities and moderately rough topography on $\mathrm{Lg}$ wave propagation at long distances and high frequencies.

\section{P-SV CASE}

To introduce the P-SV elastic screen propagator for a flat free surface, the basic idea is to incorporate plane wave reflection calculation into elastic screen method (Wu, 1994; Wu et al., 2000c). The half space is extended upward in vertical direction from free surface. The extended part has the parameters of background medium and will be used to keep records of upgoing waves which can be used for the calculation of reflected/converted waves by the free surface. The incident $\mathrm{P}$ and $\mathrm{S}$ waves at vertical profile $x=x^{\prime}$ can be decomposed into a superposition of plane waves $\mathbf{u}_{0}^{P}\left(K_{z}, x^{\prime}\right)$ and $\mathbf{u}_{0}^{S}\left(K_{z}, x^{\prime}\right)$. The propagating waves are represented by the real vertical wavenumbers, and the imaginary vertical wavenumbers correspond to the surface waves (inhomogeneous waves). Reflection at the free surface can be calculated at each step, and the total field including the reflected waves will interact with the heterogeneities. We will first treat the propagating waves (homogeneous waves) and then discuss the calculation of the fundamental mode Rayleigh wave as an example of the surface wave modeling. 


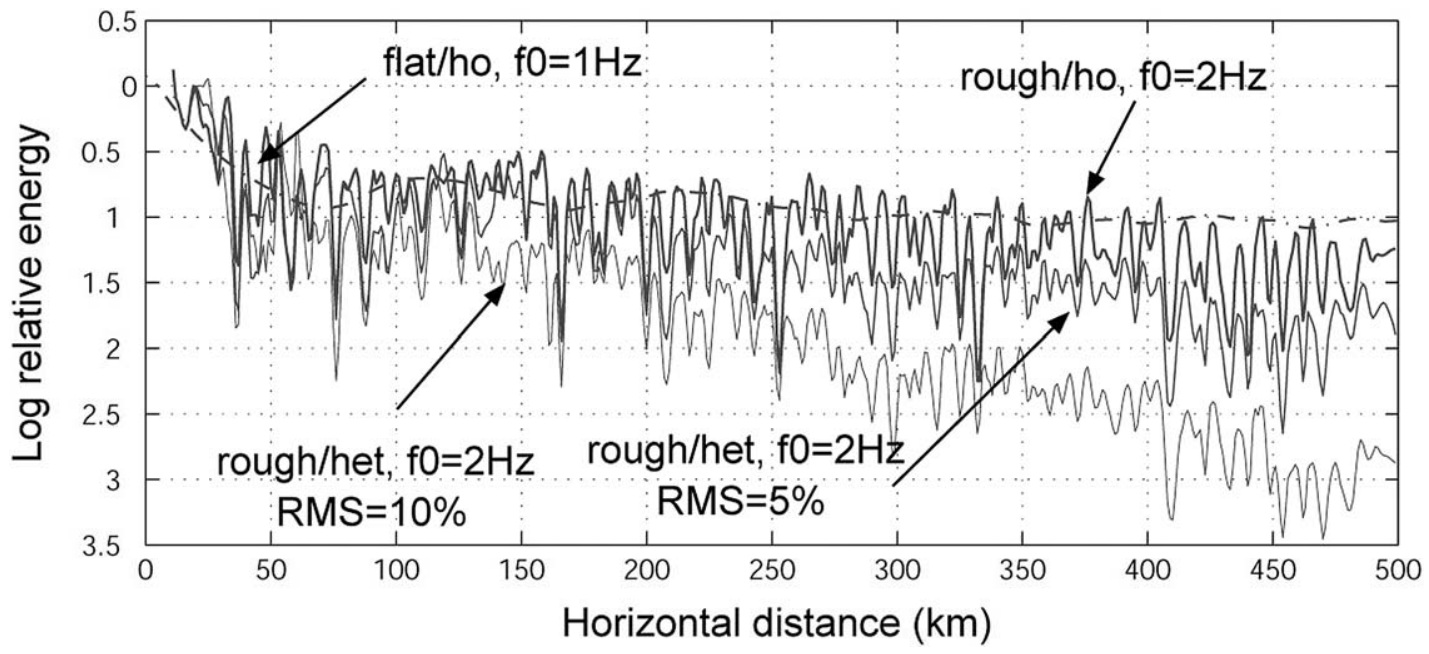

FIG. 14. Lg wave attenuation versus horizontal distances. A random medium whose correlation lengths are $6 \mathrm{~km}$ in range and $4 \mathrm{~km}$ in depth, and RMS velocity fluctuations are $5 \%$ and $10 \%$, respectively. The source is located at a depth of $8 \mathrm{~km}$, the dominant frequency $\left(f_{0}\right)$ of source time function is $2 \mathrm{~Hz}$. In the figure, "rough" means the crust with rough topography, "ho" and "het" denote homogeneous and heterogeneous crustal models, respectively. 
Applying the reflection coefficients, the free surface reflected $\mathrm{P}$ and $\mathrm{S}$ waves due to incident $\mathrm{P}$ wave can be expressed by

$$
\begin{aligned}
& \mathbf{u}^{P P}(x, z)=e^{i \gamma_{\alpha}\left(x-x^{\prime}\right)} \int \mathrm{d} K_{z}\left|\mathbf{u}_{0}^{P}\left(K_{z}, x^{\prime}\right)\right| P P \hat{\mathbf{a}}_{1} e^{-i K_{z} z}, \\
& \mathbf{u}^{P S}(x, z)=e^{i \gamma_{\alpha}\left(x-x^{\prime}\right)} \int \mathrm{d} K_{z}\left|\mathbf{u}_{0}^{P}\left(K_{z}, x^{\prime}\right)\right| P S \hat{\mathbf{a}}_{2} e^{-i K_{z}^{*} z},
\end{aligned}
$$

where $\gamma_{\alpha}=\sqrt{k_{\alpha}^{2}-K_{z}^{2}}$ is the propagating wavenumber for $\mathrm{P}$ waves (here in the $x$-direction) and $K_{z}^{*}=\sqrt{k_{\beta}^{2}-k_{\alpha}^{2}+K_{z}^{2}}$ is the transverse wavenumber of converted $\mathrm{S}$ waves determined by Snell's law. Unit vectors $\hat{\mathbf{a}}_{1}=\left(\gamma_{\alpha},-K_{z}\right) / k_{\alpha}$ and $\hat{\mathbf{a}}_{2}=\left(K_{z}^{*}, \gamma_{\alpha}\right) / k_{\beta} \cdot\left|\mathbf{u}_{0}^{P}\left(K_{z}, x^{\prime}\right)\right|$ is the scalar spectrum of the incident $\mathrm{P}$ wave with a transverse wavenumber $K_{z}$ (here in $z$-direction). The reflected $\mathrm{P}$ and $\mathrm{S}$ waves due to the incident plane $\mathrm{S}$ wave can be obtained by

$$
\begin{aligned}
& \mathbf{u}^{S P}(x, z)=e^{i \gamma_{\beta}\left(x-x^{\prime}\right)} \int \mathrm{d} K_{z}\left|\mathbf{u}_{0}^{S}\left(K_{z}, x^{\prime}\right)\right| S P \hat{\mathbf{a}}_{3} e^{-i K_{z}^{\prime *} z}, \\
& \mathbf{u}^{S S}(x, z)=e^{i \gamma_{\beta}\left(x-x^{\prime}\right)} \int \mathrm{d} K_{z}\left|\mathbf{u}_{0}^{S}\left(K_{z}, x^{\prime}\right)\right| S S \hat{\mathbf{a}}_{4} e^{-i K_{z} z},
\end{aligned}
$$

where $\gamma_{\beta}=\sqrt{k_{\beta}^{2}-K_{z}^{2}}$ is the propagating wavenumber for $\mathrm{S}$ waves (here in the $x$-direction) and $K_{z}^{\prime *}=\sqrt{k_{\alpha}^{2}-k_{\beta}^{2}+K_{z}^{2}}$ is the transverse wavenumber of the reflected $\mathrm{P}$ wave. Unit vectors $\hat{\mathbf{a}}_{3}=\left(\gamma_{\beta},-K_{z}^{\prime *}\right) / k_{\alpha}$ and $\hat{\mathbf{a}}_{4}=\left(K_{z}, \gamma_{\beta}\right) / k_{\beta}$. $\left|\mathbf{u}_{0}^{S}\left(K_{z}, x_{1}\right)\right|$ is the scalar spectrum of the incident $\mathrm{S}$ wave with a transverse wavenumber $K_{z} . P P, P S, S P$ and $S S$ in Eqs. (38)-(41) are reflection coefficients of different wave types at the free surface (Aki and Richards, 1980). Figure 15 is an example of those reflection coefficients versus horizontal slowness (ray parameter $p$ ). In Fig. $15, p_{A}$ corresponds to $\mathrm{P}$ slowness (inverse velocity) and $p_{B}$ to $\mathrm{S}$ slowness. For $p<p_{A}, \mathrm{P}$ and $\mathrm{S}$ waves are both homogeneous waves, their transverse wavenumbers are real. For $p>p_{B}, \mathrm{P}$ and $\mathrm{S}$ waves are both inhomogeneous waves, their transverse wavenumbers are imaginary. For $p_{A}<p<p_{B}$, $\mathrm{P}$ wave is inhomogeneous while $\mathrm{S}$ wave is homogeneous. A Rayleigh pole is located in the region of $p>p_{B}$. In general, we can calculate all reflected waves using Eqs. (38)-(41), once the incident fields $\left|\mathbf{u}_{0}^{P}\right|$ and $\left|\mathbf{u}_{0}^{S}\right|$ are known. However, numerically, it is more convenient to separate the calculation of Eqs. (38)-(41) into homogeneous and inhomogeneous waves, respectively.

For homogeneous waves, Eqs. (38) and (41) (common-type) can be implemented by FFT. However, the reflected waves of converted-type cannot be directly implemented by FFT because the nonlinear relationship exists between $K_{z}$ and $K_{z}^{*}$ for P-S conversion (or $K_{z}$ and $K_{z}^{\prime *}$ for S-P conversion). Although we can obtain uniform samples with respect to $K_{z}$ and $K_{z}^{*}$ (or $K_{z}$ and $K_{z}^{\prime *}$ ) by complex variable interpolation to make FFT applicable, numerical tests have shown that 

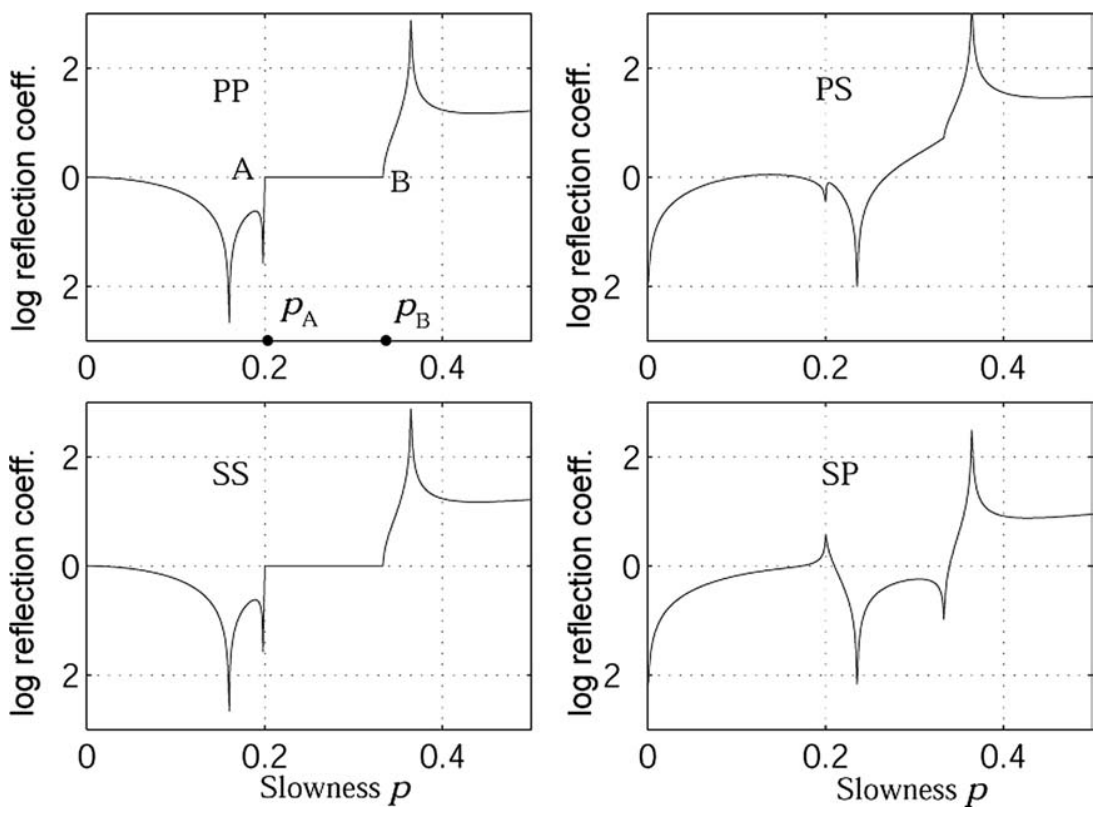

FIG. 15. The free surface reflection coefficients (in logarithmic scale) versus horizontal slowness. The $\mathrm{P}$ and $\mathrm{S}$ wave velocities for the elastic half-space are $\alpha=5 \mathrm{~km} / \mathrm{s}$ and $\beta=3.5 \mathrm{~km} / \mathrm{s}$. The $p_{A}$ and $p_{S}$ denote $\mathrm{P}$ and $\mathrm{S}$ slownesses.

the noise due to the interpolation is so strong that the accumulated errors increase very fast for multiple step propagation. In our study, the direct summations over the incident waves ( $p<p_{A}$ for $\mathrm{P}$ incidence or $p<p_{B}$ for $\mathrm{S}$ incidence) are performed for calculating the converted reflections. Figure 16 shows synthetic seismograms calculated with Eqs. (38)-(41) for an elastic half-space with only homogeneous waves. The results calculated with wavenumber integration (WI) method (dashed lines) are also shown as references. Since the source is deep compared with the propagation distance, Rayleigh wave is very weak in the exact solution. Figure 16a shows the vertical component of the displacement and Fig. 16b shows the horizontal component. From Fig. 16 we see that the calculations of the reflection and conversion by the free surface are in excellent agreement with the theory. Figure 17 shows synthetic seismograms for FloraAsnes crustal model (see, Fig. 18) using elastic screen method. A double-couple source is located at a depth of $16 \mathrm{~km}$ and has a dominant frequency of $2 \mathrm{~Hz}$. We see that both $\mathrm{P}$ and $\mathrm{S}$ waves are well excited. Figure 18 is the corresponding snapshots. From Figs. 17 and 18, the short-period phases Pn, Sn, Lg, etc., can be identified. For the elastic screen method at its current stage, only real transverse wavenumbers are used in FFT, which can only handle propagating waves (homogeneous waves). 


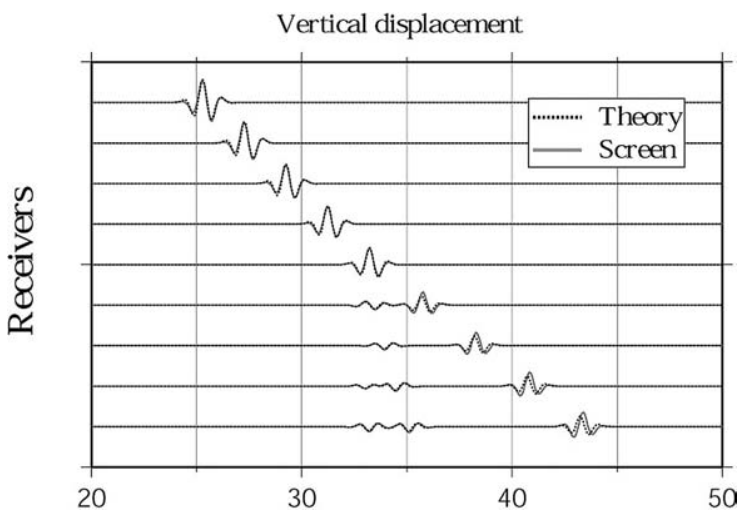

(a)

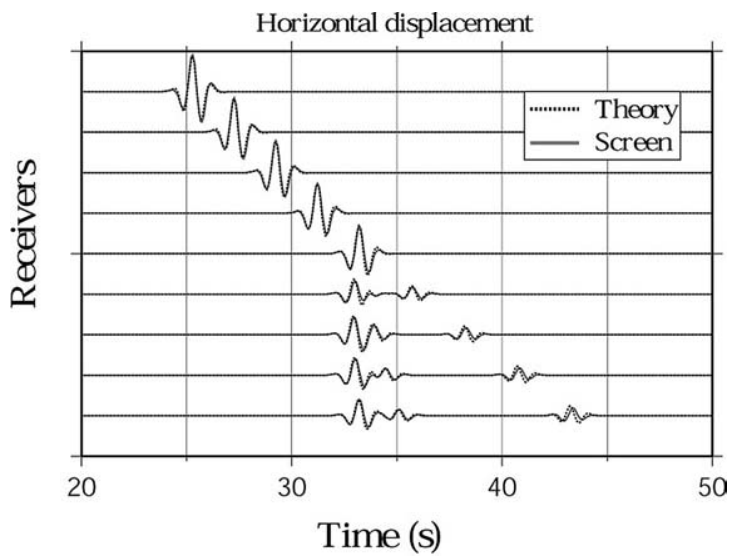

(b)

FIG. 16. Synthetic seismograms calculated by the elastic screen method (solid lines) and wavenumber integration method (dashed lines) for an elastic halfspace. Only homogeneous waves are included in the results of elastic screen method. (a) Shows the vertical components of displacement; (b) Shows the horizontal components. A point explosion source is located at the depth of $16 \mathrm{~km}$ and the dominant frequency of source time function is $1 \mathrm{~Hz}$. The first 4 receivers are placed along the free surface separated from the source by $100-124 \mathrm{~km}$, and the last 5 receivers are placed in a vertical profile at an epicenter distance of $132 \mathrm{~km}$ and with depths ranging from $0-32 \mathrm{~km}$.

For inhomogeneous waves, their transverse wavenumbers are imaginary so that Eqs. (38)-(41) cannot be calculated by FFT. However, the imaginary transverse wavenumber makes the propagation of inhomogeneous waves simple. The phase advance takes place only along the horizontal direction. It can be easily incorporated into the screen method, once the spectra of inhomogeneous waves 


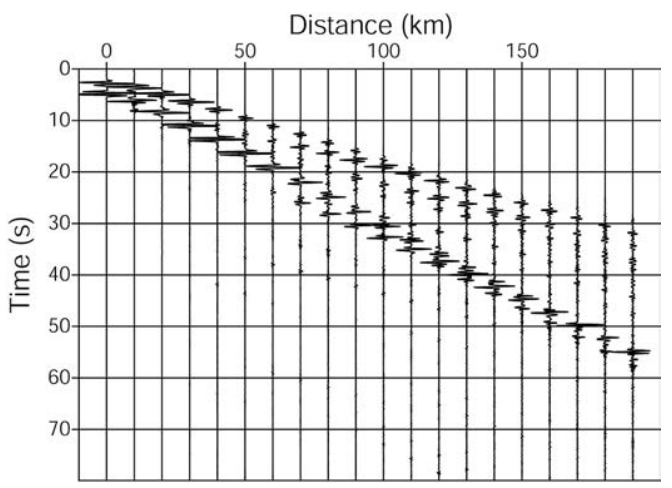

(a)

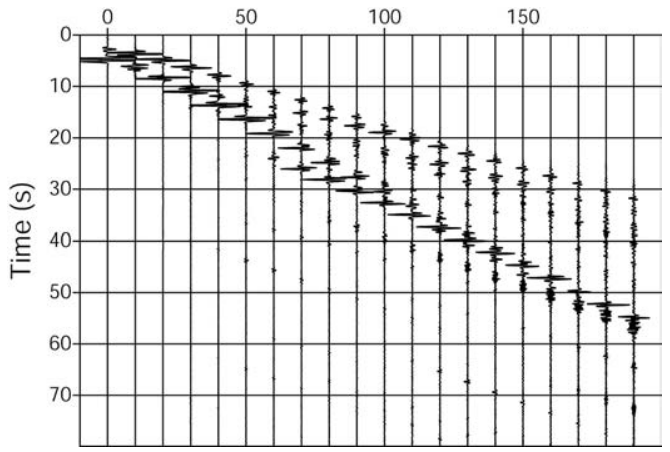

(b)

FIG. 17. Synthetic seismograms for Flora-Asnes crustal model (see Fig. 18) using P-SV elastic screen method. Only homogeneous wave are involved. (a) Shows the vertical components of displacement and (b) shows the horizontal components. A double-couple source is located at the depth of $16 \mathrm{~km}$ and has a dominant frequency of $2 \mathrm{~Hz}$. Receivers are on the surface.

are known. Another important feature of inhomogeneous waves is the exponential decay only in the direction perpendicular to propagation direction. Then the spectra of inhomogeneous waves can be calculated with Laplace transform. Figure 19 shows an example of such a treatment for Rayleigh wave propagating in homogeneous elastic half-space. The source is located at a depth of $2 \mathrm{~km}$ and has a dominant frequency of $0.5 \mathrm{~Hz}$. The vertical receiver array is located at a distance of $100 \mathrm{~km}$. Figure 19a shows the horizontal component of Rayleigh wave synthetic seismograms and Fig. 19b shows the vertical component of synthetic seismograms. The solid lines are exact solution. The agreement between the screen calculation and the exact solution is excellent. The interaction between inhomogeneous waves and heterogeneities and the conversion between body waves and surface wave are still on-going research. 

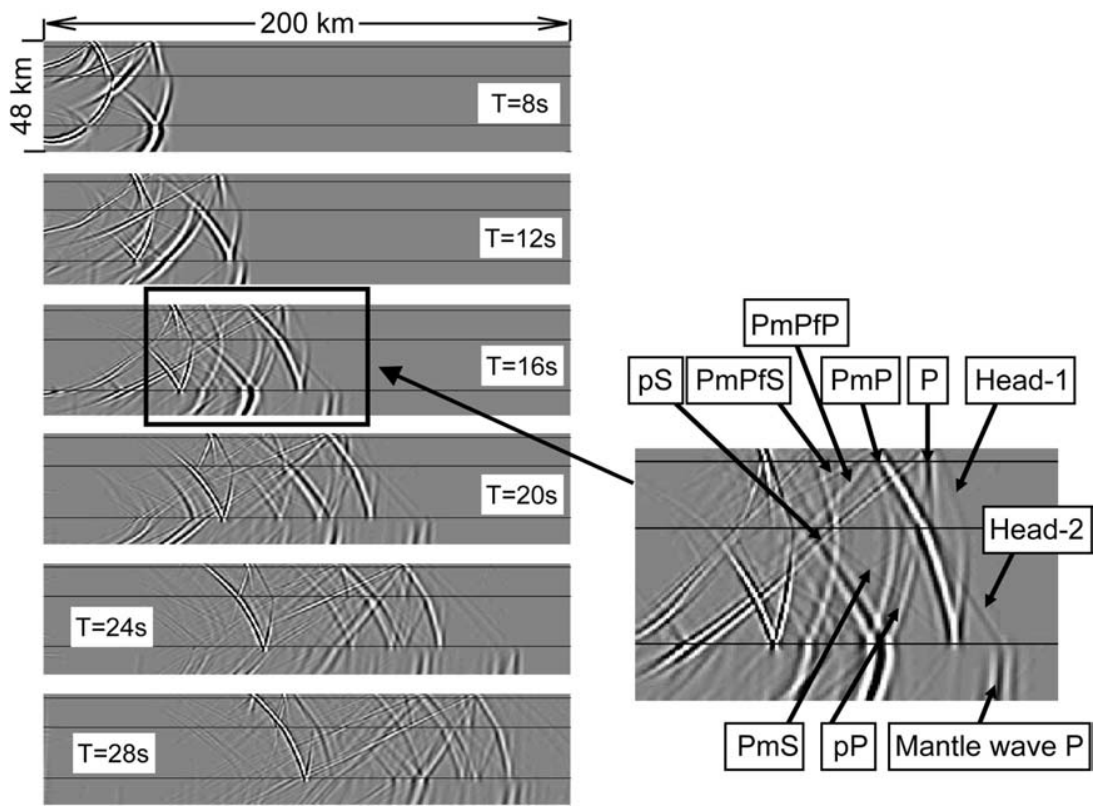

FIG. 18. Snapshots (horizontal component of displacement) for Flora-Asnes crustal model using P-SV elastic screen method. A double-couple source is located at the depth of $16 \mathrm{~km}$ and has a dominant frequency of $2 \mathrm{~Hz}$. The thicknesses of layers (from top to bottom) are $1 \mathrm{~km}, 14 \mathrm{~km}, 22 \mathrm{~km}$ and infinity, respectively. Their velocity and density parameters are $\alpha_{1}=5.2 \mathrm{~km} / \mathrm{s}, \beta_{1}=3 \mathrm{~km} / \mathrm{s}$, $\rho_{1}=2.6 \mathrm{~g} / \mathrm{cm}^{3} ; \alpha_{2}=6.0 \mathrm{~km} / \mathrm{s}, \beta_{2}=3.46 \mathrm{~km} / \mathrm{s}, \rho_{2}=2.8 \mathrm{~g} / \mathrm{cm}^{3} ; \alpha_{3}=6.51 \mathrm{~km} / \mathrm{s}$, $\beta_{3}=3.76 \mathrm{~km} / \mathrm{s}, \rho_{3}=3 \mathrm{~g} / \mathrm{cm}^{3} ; \alpha_{4}=8.05 \mathrm{~km} / \mathrm{s}, \beta_{4}=4.65 \mathrm{~km} / \mathrm{s}, \rho_{4}=3.3 \mathrm{~g} / \mathrm{cm}^{3}$. The major phases are labeled in the figure.

\section{CONCLUSION}

In the crustal waveguide environment, the major part of wave energy is carried by forward propagating waves, including forward scattered waves. Therefore, the neglect of backscattered waves in the modeling can still simulate the main features of regional waves in most cases. By neglecting backscattering in the theory, the method becomes a forward marching algorithm. A half-space screen propagator (generalized screen propagator) has been developed to accommodate the free-surface boundary condition and treat the SH wave propagation in complex crustal waveguides. The SH screen propagator has also been extended to the case of irregular surface topography by conformal or non-conformal topographic transforms. For medium sized problems, the screen-propagator method is two to three orders of magnitude faster than the finite-difference methods. 

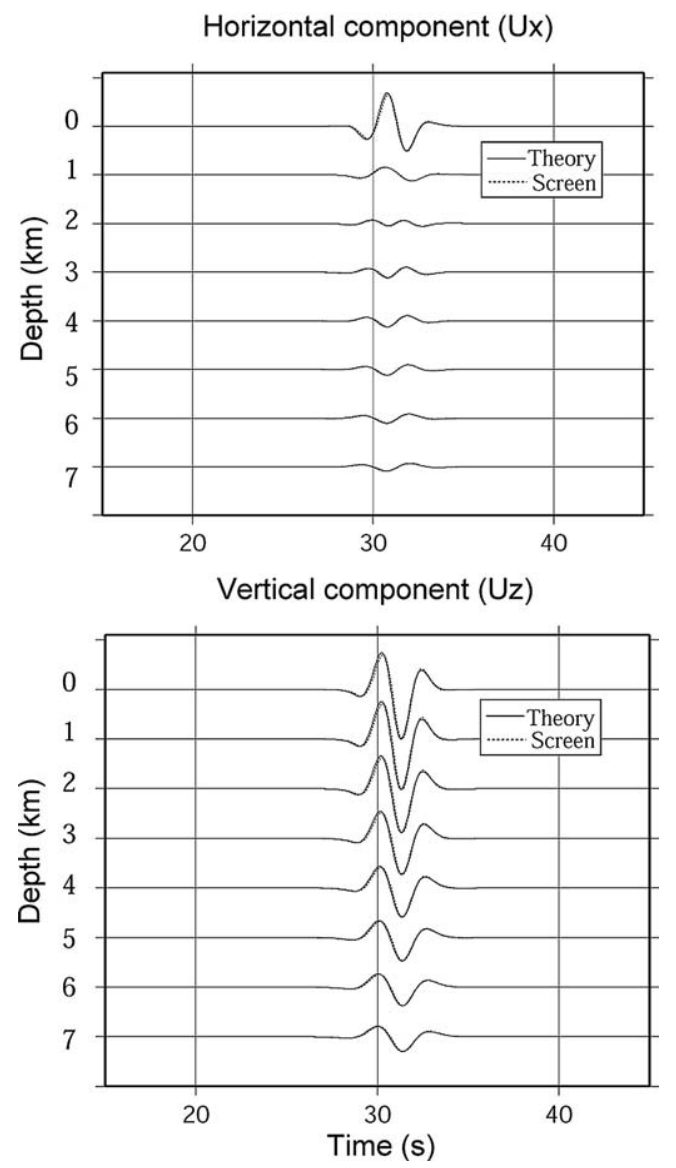

FIG. 19. Comparison of synthetic Rayleigh wave calculated using the screen method (dotted lines) with those calculated using the exact solution (solid lines). The source is located at a depth of $2 \mathrm{~km}$ and has a dominant frequency of $0.5 \mathrm{~Hz}$. (a) Shows the horizontal components of displacement of Rayleigh wave and (b) shows the vertical components. The half-space parameters are $\alpha=6 \mathrm{~km} / \mathrm{s}$ and $\beta=3.5 \mathrm{~km} / \mathrm{s}$.

In the case of P-SV elastic screen propagators, plane wave reflection calculations have been incorporated into the elastic screen method. Body waves including the reflected and converted waves can be calculated by real wavenumber integration, while surface waves (Rayleigh waves) can be obtained with imaginary wavenumber integration. Numerical tests show good agreement with the theory.

From the theoretical developments and numerical tests of both SH and P-SV screen-propagators, we see that the one-way screen propagator approach for regional wave simulation is a viable approach and the savings in computation 
time and computer storage are enormous, especially for large 3-D elastic, longrange wave propagation problems. For the SH screen propagators, the theory and method are rather complete and can treat various crustal models including large-scale structures, random heterogeneities, and irregular or rough surface topography. The method has been applied to the simulation of Lg propagation in random media and the related energy partition and attenuation. It is found that the leakage attenuation of $\mathrm{Lg}$ waves caused by large-angle forward scattering from random heterogeneities may contribute significantly to $\mathrm{Lg}$ attenuation and blockage in some regions. The apparent $Q$ for leakage attenuation as a function of normalized scale length $(\mathrm{ka})$ of the random heterogeneities agrees well with the scattering theory. More work can be done along this direction and comparison with observations may reveal the real mechanisms for Lg blockage in different regions.

More work has to be done for the P-SV problems, e.g., the conversion between body wave, guided wave and surface wave caused by crustal heterogeneities and irregular topography. Then the full 3-D elastic screen propagator will provide the full power of simulating regional wave propagation in complex crustal models.

\section{ACKNOWLEDGEMENTS}

The helpful discussions with T. Lay, S. Jin and G. Fan are greatly appreciated. This work was supported by the Department of Energy, the Defense Threat Reduction Agency and the Air Force Research Laboratory through various contracts. Facility support from the W.M. Keck Foundation is also acknowledged. Contribution number 432 of CSIDE, IGPP, University of California, Santa Cruz.

\section{REFERENCES}

Aki, K., Richards, P.G. (1980). Quantitative Seismology: Theory and Methods, vols. 1 and 2. W.H. Freeman, New York.

App, F.N., Bos, R.J., Kamm, J.R. (1996). Synthetic seismograms at regional distances for May 1995 Earthquake and Explosion source in Western China. In: Proceedings of the 18th Annual Seismic Research Symposium on Monitoring a Comprehensive Nuclear-Test-Ban Treaty. 4-6 September, pp. 119-128.

Archambeau, C., Orrey, J., Kohl, B. (1996). 3-D seismic wave synthesis and full wavefield inversion. In: Proceedings of the 18th Annual Seismic Research Symposium on Monitoring a Comprehensive Nuclear-Test-Ban Treaty. 4-6 September, pp. 129-138.

Baumgardt, D.R. (1990). Investigation of teleseismic Lg blockage and scattering using regional arrays. Bull. Seismol. Soc. Am. 80, 2261-2281.

Beillis, A., Tappert, F.D. (1979). Coupled mode analysis of multiple rough surface scattering. $J$. Acoust. Soc. Am. 66, 811-826.

Bostock, M.G., Kennett, B.L.N. (1990). The effect of 3-D structure on Lg propagation patterns. Geophys. J. Int. 101, 355-365. 
Bouchon, M., et al. (1985). Theoretical modeling of Lg wave attenuation. In: The VELA Program: A Twenty-five Year Review of Basic Research.

Bouchon, M., Barker, J. (1996). Seismic response of a hill: The example of Tarzana, California. Bull. Seismol. Soc. Am. 86, 66-72.

Bradley, C.R., Jones, E.M. (1998). Modeling propagation effects from explosion in Western China and India. In: Proceedings of the 20th Annual Seismic Research Symposium on Monitoring a Comprehensive Nuclear-Test-Ban Treaty. 21-23 September, pp. 173-181.

Bradley, C.R., Jones, L.E. (1999). Full waveform modeling of the effects of Q and structure over subregional paths in Western China. In: Proceedings of the 21st Annual Seismic Research Symposium on Monitoring a Comprehensive Nuclear-Test-Ban Treaty. 21-24 September, pp. 28-38.

Campillo, M. (1990). Propagation and attenuation characteristics of the crustal phase Lg. Pure Appl. Geophys. 132, 1-19.

Campillo, M., Paul, A. (1992). Influence of lower crustal structure on the early coda of regional seismograms. J. Geophys. Res. 97, 3405-3416.

Campillo, M., Feignier, B., Bouchon, M., Bethoux, N. (1993). Attenuation of crustal waves across the Alpine range. J. Geophys. Res. 98, 1987-1996.

Chen, X. (1990). Seismograms synthesis for multi-layered media with irregular interfaces by global generalized reflection/transmission matrices method, Part I. Theory of 2-D SH case. Bull. Seismol. Soc. Am. 80, 1696-1724.

Chen, X. (1995). Seismograms synthesis for multi-layered media with irregular interfaces by global generalized reflection/transmission matrices method, Part II. Applications of 2-D SH case. Bull. Seismol. Soc. Am. 85, 1094-1106.

Cormier, V.F., Anderson, T. (1996). Lg blockage and scattering at CNET and KEET. In: Proceedings of the 18th Annual Seismic Research Symposium on Monitoring a Comprehensive Nuclear-TestBan Treaty. 4-6 September, pp. 159-168.

Cormier, V.F., Anderson, T. (1997). Lg blockage and scattering at Central Eurasian Arrays CNET and ILPA. In: Proceedings of the 19th Annual Seismic Research Symposium on Monitoring a Comprehensive Nuclear-Test-Ban Treaty. 23-25 September, pp. 479-485.

De Wolf, D.A. (1971). Electromagnetic reflection from an extended turbulent medium: Cumulative forward-scatter single-backscatter approximation. IEEE Trans. Antennas and Propagation AP-19, 254-262.

De Wolf, D.A. (1985). Renormalization of EM fields in application to large-angle scattering from randomly continuous media and sparse particle distributions. IEEE Trans. Antennas and Propagation AP-33, 608-615.

Flatté, S.M., Dashen, R., Munk, W., Watson, K., Zachariasen, F. (1979). Sound Transmission Through a Fluctuating Ocean. Cambridge Univ. Press, Cambridge.

Fornberg, B. (1987). The pseudospectral method: Comparisons with finite differences for the elastic wave equation. Geophysics 52, 482-501.

Frankel, A. (1989). A review of numerical experiments on seismic wave scattering. In: Wu, R.S., Aki, K. (Eds.), In: Scattering and Attenuation of Seismic Waves, vol. 2. Birkhauser, Berlin, pp. 639-686.

Frankel, A., Clayton, R.W. (1986). Finite difference simulations of seismic scattering: Implications for propagation of short-period seismic waves in the crust and models of crustal heterogeneity. $J$. Geophys. Res. 91, 6465-6489.

Fu, L.Y., Wu, R.S. (2001). A hybrid BE-GS method for modeling regional wave propagation. Pure Appl. Geophys. 158, 1251-1277.

Furumura, T., Kennett, B.L.N. (1997). On the nature of regional seismic phases-II. On the influence of structural barriers. Geophys. J. Int. 129, 221-234.

Geli, L., Bard, P.Y., Jullien, B. (1988). The effects of topography on earthquake ground motion: A review and new results. Bull. Seismol. Soc. Am. 78, 42-63.

Gibson, R.L., Campillo, M. (1994). Numerical simulation of high- and low-frequency Lg-wave propagation. Geophys. J. Int. 118, 47-56. 
Goff, J., Holliger, K. (1999). Nature and origin of upper crustal seismic velocity fluctuations and associated scaling properties: Combined stochastic analyses of KTB velocity and lithology logs. J. Geophys. Res. 104, 0148-0227.

Goldstein, P., Bhattacharyya, J., Ichinose, G., Leach, R. (1999). On the sensitivity of broad band regional seismic phases to multi-dimensional earth structure: Implications for phase identification. In: Proceedings of the 21st Annual Seismic Research Symposium on Monitoring a Comprehensive Nuclear-Test-Ban Treaty. 21-24 September, pp. 58-63.

Goldstein, P., Schultz, C., Larsen, S., Minner, L. (1996). Modeling of regional wave propagation phenomena in the middle east and north Africa and new analysis capabilities in SAC2000. In: Proceedings of the 18th Annual Seismic Research Symposium on Monitoring a Comprehensive Nuclear-Test-Ban Treaty. 4-6 September, pp. 165-171.

Goldstein, P., Schultz, C., Larsen, S. (1997). The influence of deep sedimentary basins, crustal thinning, attenuation, and topography on regional phases: Selected examples from the Eastern Mediterranean and the Caspian sea regions. In: Proceedings of the 19th Annual Seismic Research Symposium on Monitoring a Comprehensive Nuclear-Test-Ban Treaty. 23-25 September, pp. 486494.

Herrmann, R.B., Mokhtar, T.A., Raoof, M., Ammon, C. (1997). Wave propagation-16 Hz to 60 s. In: Proceedings of the 19th Annual Seismic Research Symposium on Monitoring a Comprehensive Nuclear-Test-Ban Treaty. 23-25 September, pp. 495-503.

Huang, L.J., Fehler, M.C., Wu, R.S. (1999a). Extended local Born Fourier migration method. Geophysics 64, 1524-1534.

Huang, L.J., Fehler, M.C., Roberts, P.M., Burch, C.C. (1999b). Extended local Rytov Fourier migration method. Geophysics 64, 1535-1545.

Huang, L.J., Wu, R.S. (1996). 3-D prestack depth migration with acoustic pseudo-screen propagators. In: Mathematical Methods in Geophysical Imaging IV. In: Proceedings of SPIE, vol. 2822, pp. 4051.

Husebye, E.S., Ruud, B.O. (1996). Wave propagation in complex crust-CTBT implications. In: Proceedings of the 18th Annual Seismic Research Symposium on Monitoring a Comprehensive Nuclear-Test-Ban Treaty. 4-6 September, pp. 172-181.

Jih, R.S. (1996). Waveguide Effects of large-scale structural variation, anelastic attenuation, and random heterogeneity on SV Lg propagation: A finite-difference modeling study. In: Proceedings of the 18th Annual Seismic Research Symposium on Monitoring a Comprehensive Nuclear-Test-Ban Treaty. 4-6 September, pp. 182-194.

Jin, S., Wu, R.S. (1999). Depth migration with a windowed screen propagator. J. Seismic Exp. 8, 27-38.

Jin, S., Wu, R.S., Peng, C. (1999). Seismic depth migration with screen propagators. Comput. Geosci. 3, 321-335.

Jones, E.M., App, F.N., Bos, R.J. (1997). The effects of major structural features in Western China on explosion seismograms. In: Proceedings of the 19th Annual Seismic Research Symposium on Monitoring a Comprehensive Nuclear-Test-Ban Treaty. 23-25 September, pp. 504-513.

Jones, E.M., Holliger, K. (1997). Spectral analyses of the KTB sonic and density logs using robust non-parametric methods. J. Geophys. Res. 102, 18391-18403.

Keers, H., Nolet, G., Dahlen, F.A. (1996a). Ray theoretical analysis of Lg. Bull. Seismol. Soc. Am. 86, 726-736.

Keers, H., Vogfjord, G., Nolet, G., Dahlen, F.A. (1996b). High frequency propagation of crustal waves. In: Proceedings of the 18th Annual Seismic Research Symposium o Monitoring a Comprehensive Nuclear-Test-Ban Treaty. 4-6 September, pp. 199-205.

Kennett, B.L.N. (1984). Guided wave propagation in laterally varying media-I. Theoretical development. Geophys. J. R. Astron. Soc. 79, 235-255.

Kennett, B.L.N. (1986). Lg waves and structural boundaries. Bull. Seismol. Soc. Am. 76, 1133-1141.

Kennett, B.L.N. (1989). Lg-wave propagation in heterogeneous media. Bull. Seismol. Soc. Am. 79, $860-872$. 
Kennett, B.L.N. (1990). Guided wave attenuation in laterally varying media. Geophys. J. Int. 100, 415-422.

Kennett, B.L.N. (1998). Guided waves in three-dimensional structures. Geophys. J. Int. 133, 159-174.

Kennett, B.L.N., Bostock, M.G., Xie, J.K. (1990). Guided-wave tracking in 3-D: A tool for interpreting complex regional seismograms. Bull. Seismol. Soc. Am. 80, 633-642.

Kosloff, D., Kessler, D., Quieroz, A., Tessmer, E. (1990). Solution of the equations of Dynamic elasticity by a Chebychev spectral method. Geophysics $\mathbf{5 5}, \mathbf{7 3 4 - 7 4 8 .}$

Lay, T., Fan, G., Wu, R.S., Xie, X.B. (1999). Path corrections for regional phase discriminants. In: Proceedings of the 21st Annual Seismic Research Symposium on Monitoring a Comprehensive Nuclear-Test-Ban Treaty. 21-24 September, pp. 510-519.

Liu, Y.B., Wu, R.S. (1994). A comparison between phase-screen, finite difference and eigenfunction expansion calculations for scalar waves in inhomogeneous media. Bull. Seismol. Soc. Am. 84, $1154-1168$.

Maupin, V. (1989). Numerical modeling of Lg wave propagation across the North Sea central graben. Geophys. J. Int. 99, 273-283.

Maupin, V., Kennett, B.L.N. (1987). On the use of truncated model expansion in laterally varying media. Geophys. J. R. Astron. Soc. 91, 837-851.

McLaughlin, K.L., Wilkins, D. (1997). Progress in numerical methods for wave propagation. In: Proceedings of the 19th Annual Seismic Research Symposium on Monitoring a Comprehensive Nuclear-Test-Ban Treaty. 23-25 September, pp. 514-523.

Ni, J., Reese, C., Wu, J., Zhao, L.S. (1996). Crustal structure and attenuation in Southern Tibet. In: Proceedings of the 18th Annual Seismic Research Symposium on Monitoring a Comprehensive Nuclear-Test-Ban Treaty. 4-6 September, pp. 390-399.

Nolte, B., Gibson, R.L., Toksöz, M.N. (1996). Irregular-grid modeling of regional wave propagation. In: Proceedings of the 18th Annual Seismic Research Symposium on Monitoring a Comprehensive Nuclear-Test-Ban Treaty. 4-6 September, pp. 231-240.

Sato, H., Fehler, M.C. (1998). Seismic Wave Propagation and Scattering in the Heterogeneous Earth. Springer-Verlag, New York.

Schatzman, J.C. (1996). A pseudo-spectral scheme for viscoelastic seismic modeling. In: Proceedings of the 18th Annual Seismic Research Symposium on Monitoring a Comprehensive Nuclear-TestBan Treaty. 4-6 September, pp. 261-270.

Sills, L.B. (1978). Scattering of horizontally-polarized shear waves by surface irregularities. Geophys. J. R. Astron. Soc. 54, 319-348.

Wild, A.J., Hudson, J.A. (1998). A geometrical approach to the elastic complex screen. J. Geophys. Res. 103, 707-725.

Wu, R.S. (1982). Attenuation of short period seismic wave due to scattering. Geophys. Res. Lett. 9, 9-12.

Wu, R.S. (1994). Wide-angle elastic wave one-way propagation in heterogeneous media and an elastic wave complex-screen method. J. Geophys. Res. 99, 751-766.

Wu, R.S. (1996). Synthetic seismograms in heterogeneous media by one-return approximation. Pure Appl. Geophys. 148, 155-173.

Wu, R.S., Aki, K. (Eds.) (1988). Scattering and Attenuation of Seismic Waves, vol. I. Birkhauser Verlag, Boston.

Wu, R.S., Aki, K. (Eds.) (1989). Scattering and Attenuation of Seismic Waves, vol. II. Birkhauser Verlag, Boston.

Wu, R.S., Aki, K. (Eds.) (1990). Scattering and Attenuation of Seismic Waves, vol. III. Birkhauser Verlag, Boston.

Wu, R.S., Huang, L.J. (1995). Reflected wave modeling in heterogeneous acoustic media using the de Wolf approximation. In: Mathematical Methods in Geophysical Imaging-III. In: Proceedings of SPIE, vol. 2571, pp. 176-186. 
Wu, R.S., Huang, L.J., Xie, X.B. (1995). Backscattered wave calculation using the De Wolf approximation and a phase-screen propagator. In: SEG 65th Annual Meeting, pp. 1293-1296. Expanded Abstracts.

Wu, R.S., Jin, S., Xie, X.B. (1996). Synthetic seismograms in heterogeneous crustal waveguides using screen propagators. In: Proceedings of the 18th Annual Seismic Research Symposium on Monitoring a Comprehensive Nuclear-Test-Ban Treaty. 4-6 September, pp. 291-300.

Wu, R.S., Jin, S., Xie, X.B. (2000a). Seismic wave propagation and scattering in heterogeneous crustal waveguides using screen propagators: I SH waves. Bull. Seismol. Soc. Am. 90, 401-413.

Wu, R.S., Jin, S., Xie, X.B. (2000b). Energy partition and attenuation of Lg waves by numerical simulations using screen propagators. Phys. Earth Planet. Int. 120, 227-243.

Wu, R.S., Jin, S., Xie, X.B., Lay, T. (1997). Verification and applications of GSP (Generalized screen propagators) method for regional wave propagation. In: Proceeding of 19th Annual Seismic Research Symposium on Monitoring a Comprehensive Nuclear-Test-Ban Treaty, pp. 552-561.

Wu, R.S., Xie, X.B. (1994). Multi-screen backpropagator for fast 3-D elastic prestack migration. In: Mathematical Methods in Geophysical Imaging-II. In: Proceedings of SPIE, vol. 2301, pp. 181193.

Wu, R.S., Xie, X.B., Jin, S., Fu, L., Lay, T. (1998). Seismic wave propagation and scattering in heterogeneous crustal waveguides using screen propagators. In: Proceedings of the 20th Annual Seismic Research Symposium on Monitoring a Comprehensive Nuclear-Test-Ban Treaty. 21-23 September, pp. 201-210.

Wu, R.S., Xie, X.B., Wu, X.Y. (1999). Lg wave simulations in heterogeneous crusts with irregular topography suing half-space screen propagators. In: Proceedings of the 21st Annual Seismic Research Symposium on Monitoring a Comprehensive Nuclear-Test-Ban Treaty. 21-24 September, pp. 683-693.

Wu, R.S., Xie, X.B., Wu, X.Y. (2000c). Lg wave propagation using SH and P-SV screen propagators in heterogeneous crusts with irregular topography. In: Proceedings of the 22nd Annual Seismic Research Symposium on Monitoring a Comprehensive Nuclear-Test-Ban Treaty.

Wu, R.S., Xu, Z., Li, X.P. (1994). Heterogeneity spectrum and scale-anisotropy in the upper crust revealed by the German continental deep-drilling (KTB) holes. Geophys. Res. Lett. 21, 911-914.

Wu, X.Y., Wu, R.S. (1999). Wide-angle thin-slab propagator with phase matching for elastic wave modeling. In: SEG 69th Annual Meeting, pp. 1867-1870. Expanded Abstracts.

Wu, X.Y., Wu, R.S. (2001). Lg wave simulation in heterogeneous crusts with surface topography using screen propagators. Geophys. J. Int. 146, 670-678.

Wu, X.Y., Wu, R.S. (2005). AVO modeling using elastic thin-slab method. Geophysics, in press.

Xie, J. (1993). Simultaneous inversion for source spectrum and path Q using Lg with application to three Semipalatinsk explosions. Bull. Seismol. Soc. Am. 83, 1547-1562.

Xie, J., Mitchell, B.J. (1991). Lg coda and $Q$ across Eurasia. In: Mitchell, B.J. (Ed.), Yield and Discrimination Studies in Stable Continental Regions. Phillips Laboratory, Hanscom Air Force Base, MA, pp. 77-91. Report PL-TR-91-2286.

Xie, X.B., Ge, Z., Lay, T. (2005). Investigating explosion source energy partitioning and Lg-wave excitation using a finite-difference plus slowness analysis method. Bull. Seismol. Soc. Am. 95, 2412-2427.

Xie, X.B., Mosher, C.C., Wu, R.S. (2000). The application of wide-angle screen propagator to 2-D and 3-D depth migrations. In: 70th Annual International Meeting, SEG, pp. 878-881. Expanded Abstracts.

Xie, X.B., Lay, T. (1994). The excitation of explosion Lg, a finite-difference investigation. Bull. Seismol. Soc. Am. 84, 324-342. Expanded Abstracts.

Xie, X.B., Wu, R.S. (1995). A complex-screen method for modeling elastic wave reflections. In: $S E G$ 65th Annual Meeting, pp. 1269-1272. Expanded Abstracts.

Xie, X.B., Wu, R.S. (1996). 3-D elastic wave modeling using the complex screen method. In: SEG 66th Annual Meeting, pp. 1247-1250. Expanded Abstracts. 
Xie, X.B., Wu, R.S. (1998). Improving the wide angle accuracy of the screen method under large contrast. In: SEG 68th Annual Meeting, pp. 1811-1814. Expanded Abstracts.

Xie, X.B., Wu, R.S. (1999). Improving the wide angle accuracy of the screen propagator for elastic wave propagation. In: SEG 69th Annual Meeting, pp. 1863-1866. Expanded Abstracts.

Xie, X.B., Wu, R.S. (2001). Modeling elastic wave forward propagation and reflection using the complex-screen method. J. Acoust. Soc. Am. 109, 2629-2635.

Xie, X.B., Wu, R.S. (2005). Multicomponent prestack depth migration using elastic screen method. Geophysics 70, S30-S37. 